\title{
Synthesis of Nanoscale Heterostructures Comprised of Metal Nanowires, Carbon Nanotubes, and Metal Nanoparticles: Investigation of Their Structure and Electrochemical Properties
}

\author{
Nitin Chopra, ${ }^{1,2}$ Junchi Wu, $^{1}$ and Paaras Agrawal ${ }^{3}$ \\ ${ }^{1}$ Metallurgical and Materials Engineering, Center for Materials for Information Technology (MINT), \\ The University of Alabama, P.O. Box 870202, Tuscaloosa, AL 35401, USA \\ ${ }^{2}$ Department of Biological Sciences, The University of Alabama, P.O. Box 870202,Tuscaloosa, AL 35401, USA \\ ${ }^{3}$ NSF-REH, Northridge High School, 2901 Northridge Road, Tuscaloosa, AL 35406, USA
}

Correspondence should be addressed to Nitin Chopra; nchopra@eng.ua.edu

Received 18 November 2014; Accepted 30 December 2014

Academic Editor: Miguel A. Correa-Duarte

Copyright (C) 2015 Nitin Chopra et al. This is an open access article distributed under the Creative Commons Attribution License, which permits unrestricted use, distribution, and reproduction in any medium, provided the original work is properly cited.

\begin{abstract}
One-dimensional nanoscale heterostructures comprised of multisegment gold-nickel nanowires, carbon nanotube, and nickel nanoparticles were fabricated in a unique approach combining top-down and bottom-up assembly methods. Porous alumina template was utilized for sequential electrodeposition of gold and nickel nanowire segments. This was followed by chemical vapor deposition growth of carbon nanotubes on multisegment gold-nickel nanowires, where nickel segment also acted as a carbon nanotube growth catalyst. The aligned arrays of these gold-nickel-carbon nanotube heterostructures were released from porous alumina template and then subjected to wet-chemical process to be decorated with nickel/nickel oxide core/shell nanoparticles. $\mathrm{X}$-ray diffraction, scanning electron microscopy, transmission electron microscopy, and Raman spectroscopy were utilized for morphology, interface, defect, and structure characterization. The electrochemical performance of these heterostructures was studied using cyclic voltammetry method and the specific capacitance of various heterostructures was estimated and compared.
\end{abstract}

\section{Introduction}

Multicomponent nanomaterials can surpass the challenges of multifunctionality associated with single-component nanostructures, where the former will introduce synergistic effects by way of morphology, composition, and properties of individual component in nanoscale heterostructures $[1,2]$. Towards this end, metallic nanowire-carbon nanotube (CNT) heterostructures attract research attention because of their unique properties, controlled growth, and ability to selfassemble. For example, these multisegment or axially heterostructured nanowires fabricated by deposition inside porous alumina (AAO) template can result in segmentspecific hydrophilicity or hydrophobicity allowing for novel self-assembly route [3]. In regard to growth control, both nanowires and CNTs can be grown on desired substrates with controlled sizes and spatial arrangements [4-6]. In this regard, AAO is also a suitable hard template route that allows for both diameter and site density (number of nanowires/
CNTs per unit area) control depending upon the pore size and density of AAO. This approach has a potential to combine multiple processing steps to integrate metal nanowires with CNTs.

One-dimensional metal-CNT heterojunctions with Ohmic or Schottky current-voltage $(I-V)$ characteristic are building blocks in devices, as planar metal-semiconductor heterojunctions are building blocks in microelectronics [7]. A report demonstrates synthesis of ordered vertical arrays of nickel- (Ni-) multiwalled CNT (MWCNT-) amorphous CNT (a-CNT) heterojunctions, which consist of a Ni nanowire, a MWCNT, and an a-CNT connected end to end (axial heterostructure), where the MWCNT is semiconducting and two Schottky contacts exist at the two ends of the MWCNT [8]. The $I-V$ characteristics of the heterojunctions embedded in the arrays were measured and analyzed by a conductive AFM. It was observed that the Schottky contacts in the heterojunctions resulted in rectifying $I-V$ characteristics. 


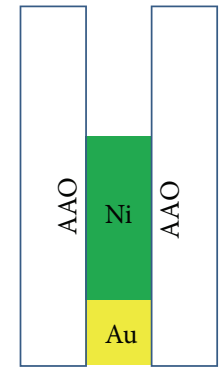

(a)

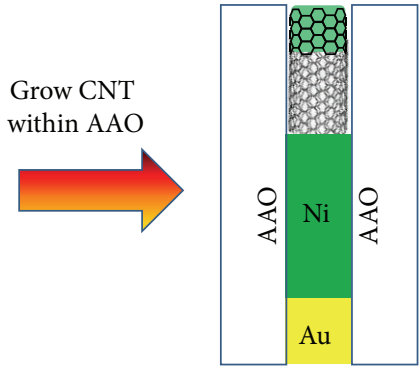

(b)

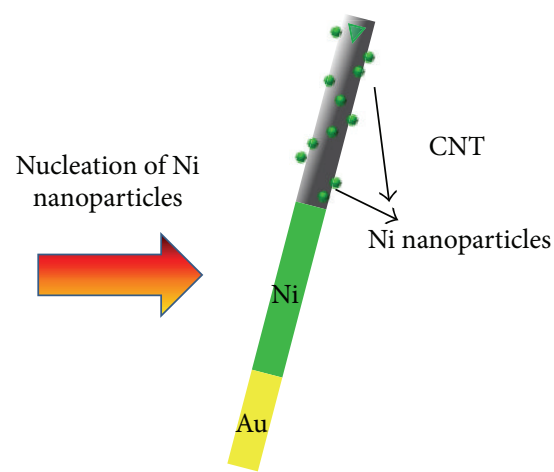

(c)

FIGURE 1: Schematic illustrating (a) electrodeposition of Au/Ni nanowire, (b) growth of CNT on tip of nanowire in a CVD process, and (c) decoration of $\mathrm{Ni} / \mathrm{NiO}$ core/shell nanoparticles on NW-CNT heterostructure in a wet-chemical approach.

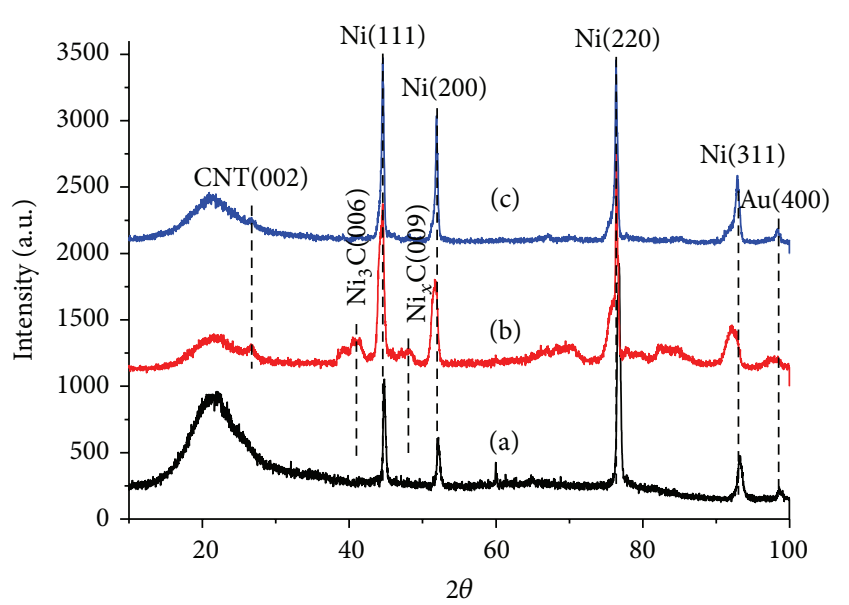

FIgURE 2: XRD of (a) Au/Ni NWs, (b) NW-CNT heterostructures, and (c) NW-CNC heterostructures.

CNTs have been used as electrodes for supercapacitors because of their excellent electrical properties and high surface areas $[9,10]$. However, CNT based supercapacitors have their own problems [11]. One of the major issues is the high contact resistance between the electrode and the current collector which limits their performance [10]. This contact resistance could be lowered by growing CNTs directly onto a conductive substrate, which can act as a current collector. Thus, a hybrid metal-CNT heterostructure may reduce the contact resistance. A recent work shows the fabrication of ultra-high power supercapacitors by using multisegmented CNT-Au nanowire structures as electrode, where both the $\mathrm{CNT}$ electrode and current collectors (Au nanowires) are integrated into an axially heterostructured system [12].

While CNT can store energy as electrochemical doublelayer capacitor, another type of supercapacitors called redox supercapacitor or pseudocapacitor, which utilizes reversible Faradaic-type charge transfer for charge/discharge process, has also been extensively investigated [13]. Nickel oxide (NiO) is a good candidate for surface redox reactions due to its high specific capacity, low cost, and good environmental compatibility [14]. However, the high resistivity of $\mathrm{NiO}$ electrodes is a serious drawback to their practical applications to supercapacitors [15]. An approach to improve the performance of $\mathrm{NiO}$ electrode is to combine carbon materials (doublelayer capacitor) and $\mathrm{NiO}$ (pseudocapacitor) as supercapacitor $[15,16]$. Herein, we reported the fabrication of three different nanocomposites consisting of $\mathrm{Au} / \mathrm{Ni}$ nanowires (NWs), nanowires with CNTs (NW-CNT heterostructures), and nanowires and CNTs decorated with $\mathrm{Ni} / \mathrm{NiO}$ core/shell nanoparticles (NW-CNC heterostructures), respectively. The multisegment nanowires were prepared by electrodeposition in AAO template, CNTs were grown on nanowires within AAO templates in a CVD method, and $\mathrm{Ni}$ nanoparticles were nucleated onto heterostructures in a wet-chemical method. This multistep process of fabricating complex metal nanowire-CNT-nanoparticle heterostructures is easy to control and suitable for production in large quantity. The produced nanostructures or heterostructures at each step of fabrication were thoroughly studied for their morphology, size, structure, and interfaces using microscopic and spectroscopic methods. The capacitance of the produced nanostructures and heterostructures was compared and analyzed using cyclic voltammetry $(\mathrm{CV})$ measurement.

\section{Experimental Section}

2.1. Material and Methods. Nickel sulfate hexahydrate $\left(\mathrm{NiSO}_{4} \cdot 6 \mathrm{H}_{2} \mathrm{O}\right)$ was purchased from Mallinckrodt Baker, Inc. (New Jersey). Gold (I) potassium cyanide, $\left(\mathrm{KAu}(\mathrm{CN})_{2}\right)$, sodium hydrogen carbonate $\left(\mathrm{NaHCO}_{3}, 99.7 \sim 100.3 \%\right)$, and tri-n-octylphosphine oxide (TOPO, 98\%) were bought from Alfa Aesar (Ward Hill, MA). Sodium hydroxide $(\mathrm{NaOH})$ and sodium chloride $(\mathrm{NaCl})$ were purchased from Fisher Scientific (New Jersey). Potassium hydroxide (KOH) was bought from Acros Organics (New Jersey). Nickel acetate tetrahydrate $\left(\left(\mathrm{CH}_{3} \mathrm{CO}_{2}\right)_{2} \mathrm{Ni} \cdot 4 \mathrm{H}_{2} \mathrm{O}, 98 \%\right)$, oleylamine $\left(\mathrm{C}_{18} \mathrm{H}_{37} \mathrm{~N}\right)$, and trioctylphosphine (TOP, 90\%) were purchased from Sigma-Aldrich (St. Louis). All chemicals were used without further purification. Labnet centrifuge (Edison, NJ) was used to wash and separate samples. Before characterization, samples were dried and stored in a VWR vacuum oven (West Chester, PA). DI water $(18.0 \mathrm{M} \Omega-\mathrm{cm})$ 

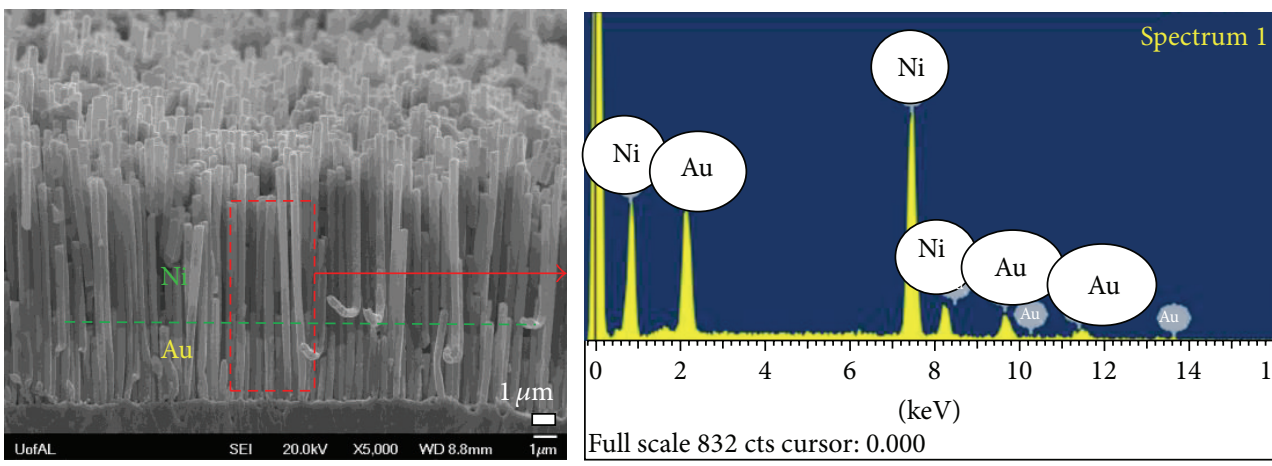

16 \begin{tabular}{cc}
\hline Element & Atomic \% \\
\hline $\mathrm{Ni} \mathrm{K}$ & 88.68 \\
$\mathrm{Au} \mathrm{M}$ & 11.32 \\
\hline
\end{tabular}

(a)

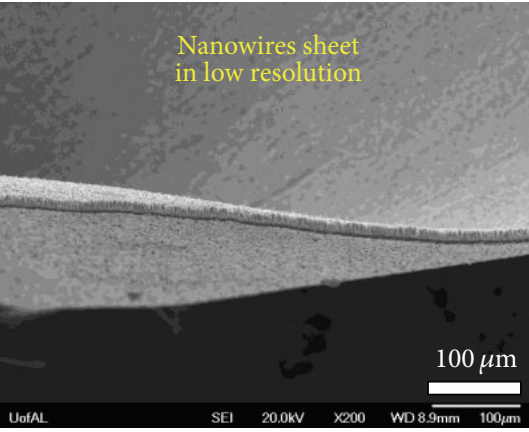

(b)

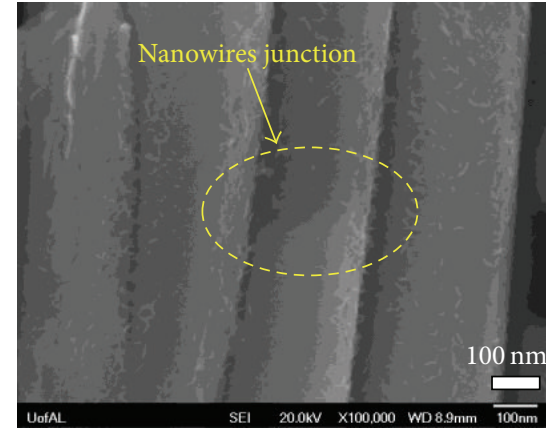

(c)

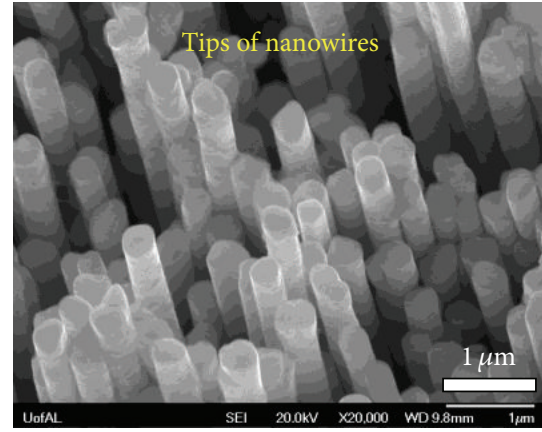

(d)

FIgURE 3: (a) SEM image of vertically aligned Au/Ni NWs with EDS showing elemental composition. (b) SEM image showing the Au/Ni NWs over a large area after being released from porous template. (c) Nanowire heterojunctions and (d) tips.

was obtained from a Barnstead International DI water system (E-pure D4641). Electrodeposition was performed using electrochemical workstation Parastat 2273 (Princeton Applied Research). CNT growth processes were conducted in a Lindberg blue 3-zone tube furnace (Watertown, WI).

\subsection{Synthesize Multisegment Metal Nanowires (NWs). A thin} layer of $\mathrm{Au} / \mathrm{Ag}$ ( $\sim 5 \mathrm{~nm}$ thick) was sputtered on the back side of porous alumina (AAO, pore size $\sim 200 \mathrm{~nm}$ ) as the conductive part of working electrode. The working electrode was connected with electrochemical station using copper wire and silver paint. Au nanowires were potentiostatistically deposited from $0.02 \mathrm{M}$ gold potassium cyanide in $\mathrm{pH}=10.0$ $\mathrm{NaHCO}_{3} / \mathrm{NaOH}$ buffer solution, while $\mathrm{Ni}$ nanowires were potentiostatistically deposited using sulfate solution with boric acid adjusting $\mathrm{pH}$. Two-segment nanowires ( $\mathrm{Au} / \mathrm{Ni}$ nanowires or also referred to as "NWs") were obtained by subsequently deposition of each segment from the corresponding electrolyte. The whole processes were performed at room temperature. After electrodeposition, AAO templates with nanowires were washed with DI water and dried in vacuum oven.

2.3. Carbon Nanotube (CNT) Growth on Nanowires (NWCNT Heterostructures). After reducing oxidized Ni in hydrogen, CNTs were grown on the tip of Au-Ni nanowires in a CVD process, involving xylene as a carbon source. Pure xylene precursor was injected through a syringe injector into a preheated zone for $\sim 2 \mathrm{~min}$ and subsequently transported into the reaction zone $\left(\sim 700^{\circ} \mathrm{C}\right)$ inside the quartz tube furnace. The xylene flow rate was reduced to $5 \mathrm{~mL} / \mathrm{h}$ when $\mathrm{H}_{2}$ in Ar carrier gas was introduced in the CVD reactor, where $\mathrm{H}_{2}$ acted as an oxygen scavenger. The CVD reaction was continued for $1 \mathrm{~h}$, after which $\mathrm{H}_{2}$ and xylene flow were discontinued and furnace was cooled down under Ar flow. After the growth of CNT, AAO template was removed away by $3 \mathrm{M} \mathrm{KOH}$ and the obtained NW-CNT heterostructures were washed with DI water and stored in vacuum oven for the next step.

2.4. Nucleation of Ni Nanoparticles on NW-CNT Heterostructures (NW-CNC Heterostructures). The NW-CNT heterostructures were decorated with $\mathrm{Ni}$ nanoparticles through a direct nucleation process [17]. After being removed from AAO template, the brush-like NW-CNT heterostructures were premixed with nickel acetate and oleylamine in a three-neck round bottom flask. The mixture was heated in $\sim 90^{\circ} \mathrm{C}$ oil bath under $\mathrm{N}_{2}$ atmosphere for $40 \mathrm{~min}$, followed by adding TOPO and TOP as stabilizer for Ni nanoparticle nucleation and growth. The temperature was increased from $90^{\circ} \mathrm{C}$ to $250^{\circ} \mathrm{C}$ at rate of $10^{\circ} \mathrm{C} / \mathrm{min}$ and then held at $250^{\circ} \mathrm{C}$ for $30 \mathrm{~min}$. After reaction, the product was washed several times with a mixture of hexane and acetone and then dried in vacuum oven overnight. The resulting product is referred to as NW-CNC heterostructures.

2.5. Cyclic Voltammetry Measurement. Cyclic voltammetry (CV) was used to measure the capacitance for NWs, NW$\mathrm{CNT}$, and NW-CNC heterostructures in $9 \mathrm{M}, 6 \mathrm{M}, 3 \mathrm{M}, 1 \mathrm{M}$, 


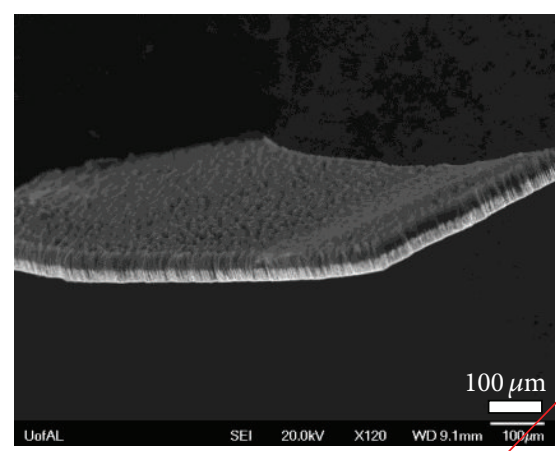

(a)
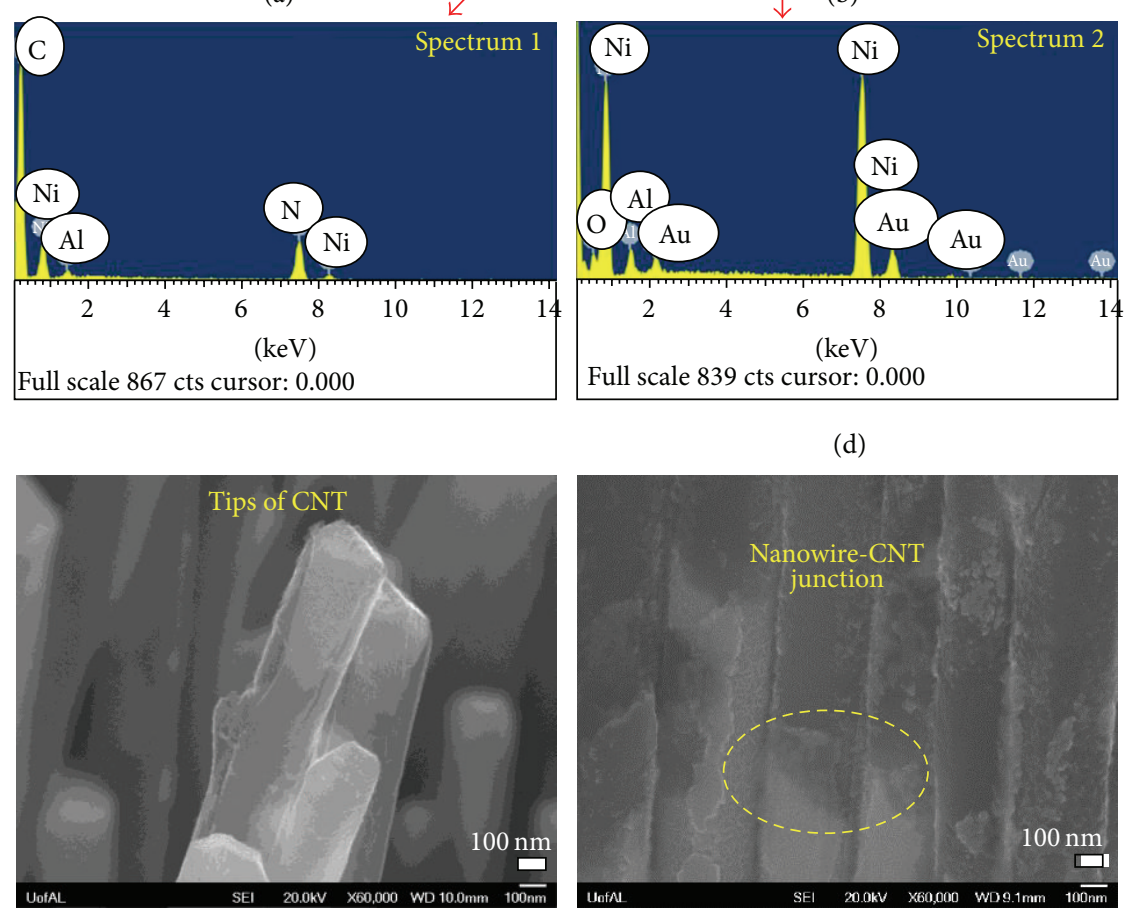

(e) (d)

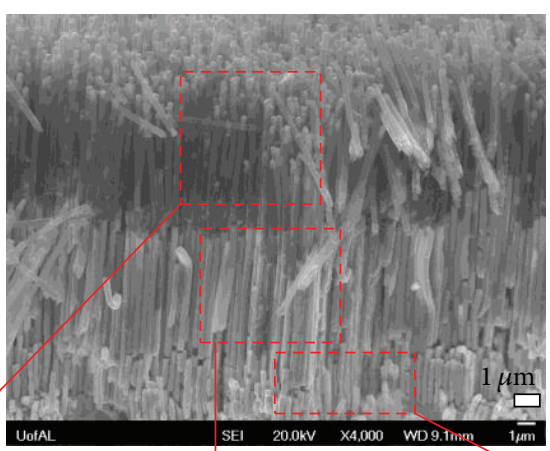

(b)

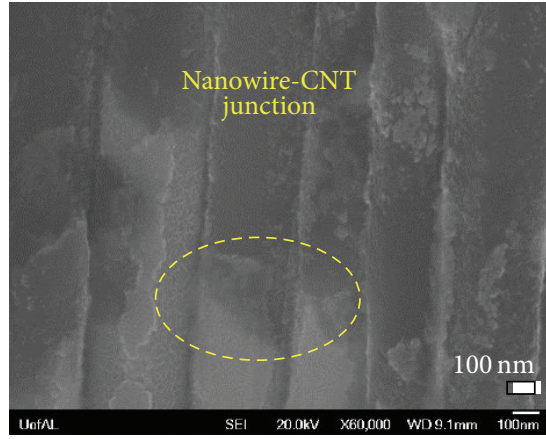

(f)

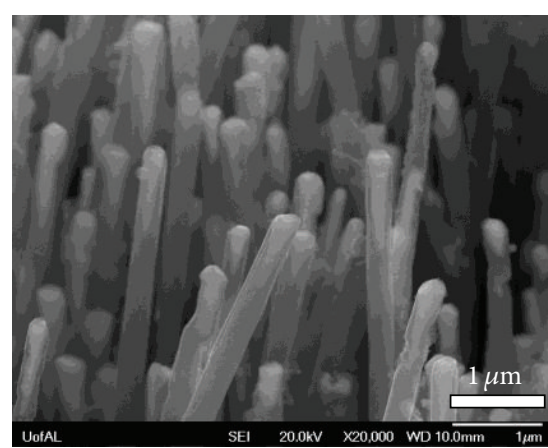

(c)
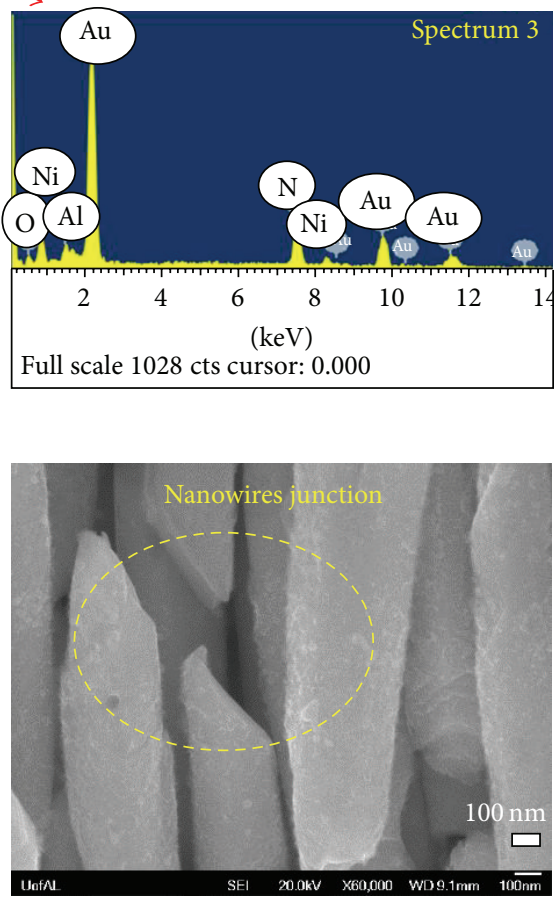

(g)

FIGURE 4: SEM images and EDS for NW-CNT heterostructures. (a) Vertically aligned array of NW-CNT heterostructures over a large area substrate after being released from porous template. (b) Various segments of heterostructures analyzed using EDS. (c) CNT segment for the array shown in (a). (e) CNT tips with catalyst Ni nanoparticle within the core. (f) Ni nanowire segment and CNT junctions. (g) Disconnected nanowire segments after the CVD growth process.

and $0.05 \mathrm{M} \mathrm{KOH}$, respectively. To protect the tip (vycor) of $\mathrm{Ag} / \mathrm{AgCl}$ reference electrode from being dissolved in base solution, reference electrode was immersed in a saturated $\mathrm{KCl}$ solution and an agar salt bridge was used to connect two solutions. Platinum wire was used as counter electrode. Copper wires were used conductor for electrode. Insulating paint was used to passivate the back face of working electrode and the exposed part of copper wire. CV measurements were conducted within -1.2 and $0.4 \mathrm{~V}$ versus the $\mathrm{Ag} / \mathrm{AgCl}$ at different scan rate as $15 \mathrm{mV} / \mathrm{s}, 30 \mathrm{mV} / \mathrm{s}$, and $50 \mathrm{mV} / \mathrm{s}$, respectively. With each scan rate, $\mathrm{CV}$ measurements were repeatedly scanned for 10 times.

2.6. Characterization Methods. Scanning electron microscopy (SEM) images energy-dispersive X-ray spectra (EDS) were obtained using FE-SEM JEOL-7000 at $20 \mathrm{kV}$. Tecnai
F-20 was used to collect transmission electron microscopy (TEM) images and EDS line-profiling at $200 \mathrm{kV}$. TEM samples were prepared by dispersing samples on lacey carbon TEM copper grids purchased from Ted Pella Inc. (Redding, $\mathrm{CA})$. The nanowire/CNT diameter/length and average size of $\mathrm{Ni}$ nanoparticles were measured form TEM and SEM images, where more than 200 nanowires/CNTs/nanoparticles were counted and measured. All the measurements were carried out using Adobe Photoshop software. X-ray diffraction (XRD) data of samples were recorded with a Philips diffractometer (XRG 3100, $\mathrm{Cu} \mathrm{Ka}$ radiation, $35 \mathrm{~mA}$ and $40 \mathrm{kV}$ ). Raman spectra and chemical mapping of samples were acquired using a Bruker Senterra Raman microscope (Bruker Optics Inc., Woodlands, TX) with $785 \mathrm{~nm}$ laser source at $10-25 \mathrm{~mW}$ laser power and 50x objective lens. The Raman mapping acquisition conditions were set at 


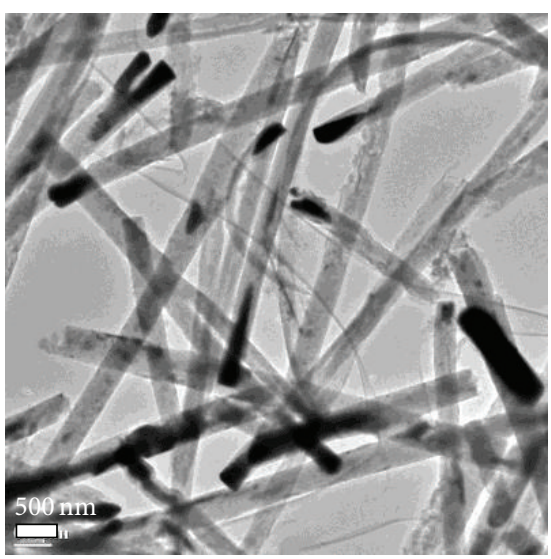

(a)

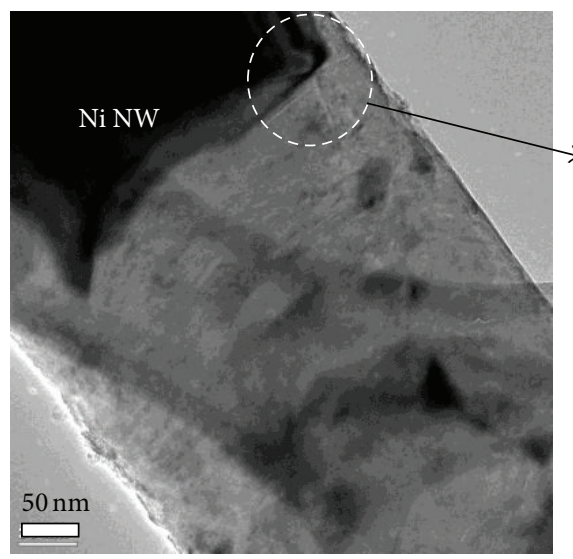

(d)

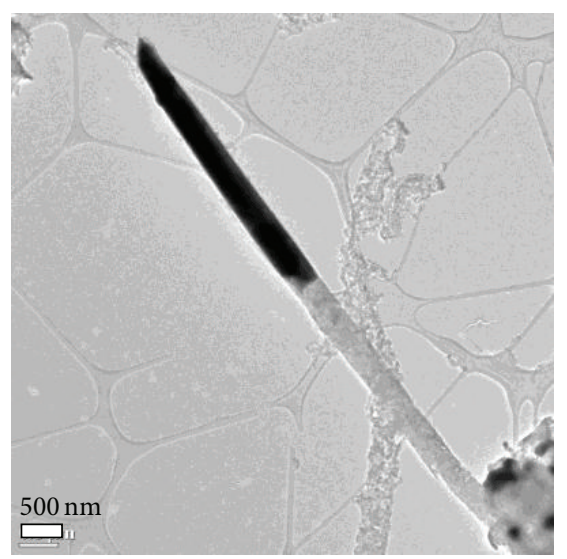

(b)

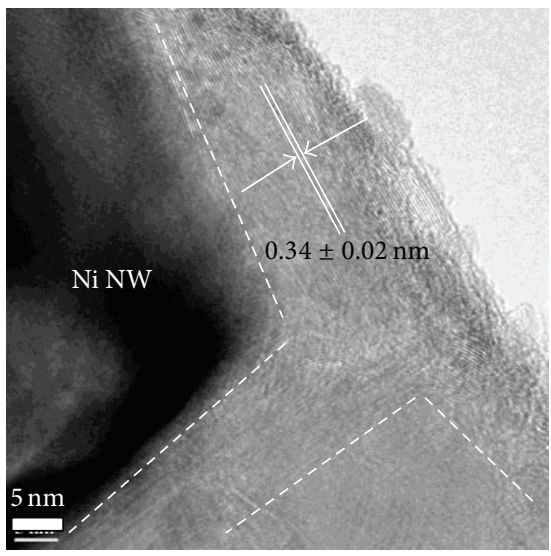

(e)

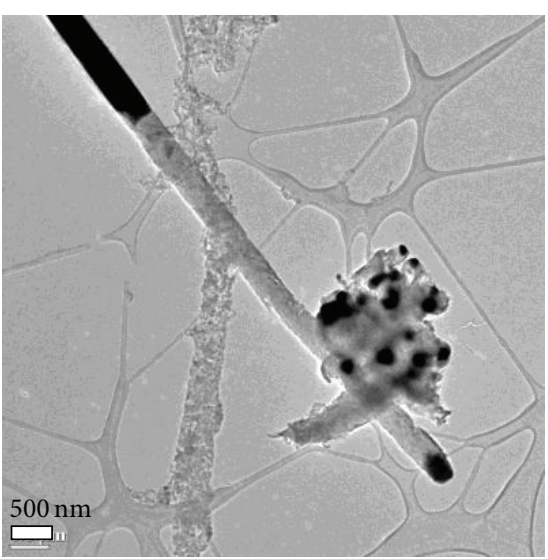

(c)

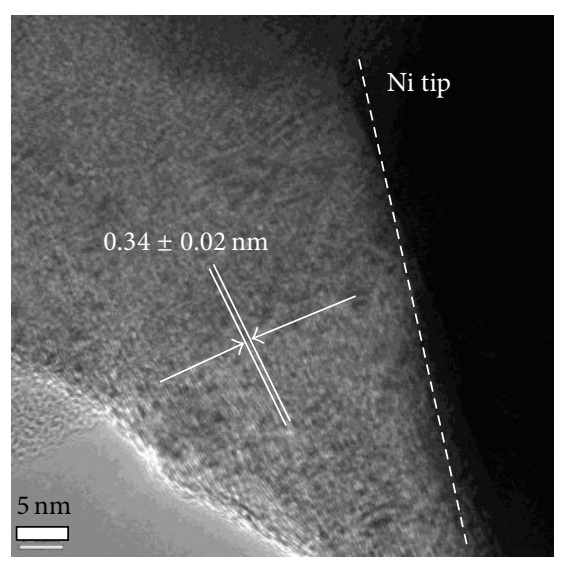

(f)

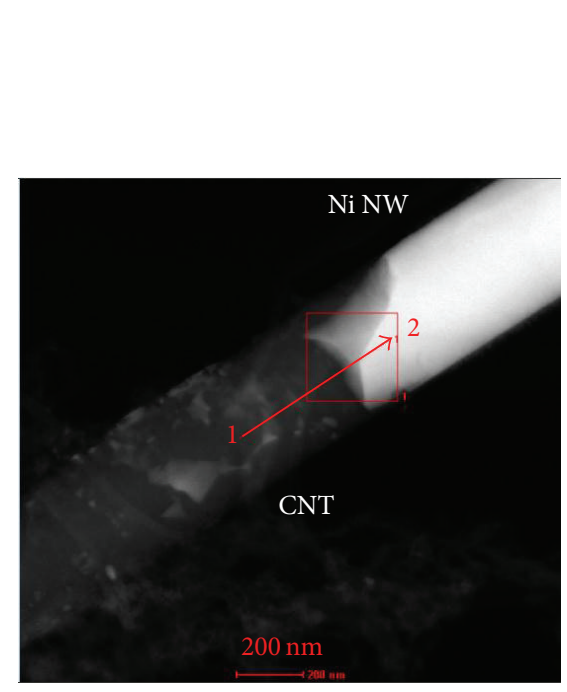

(g)

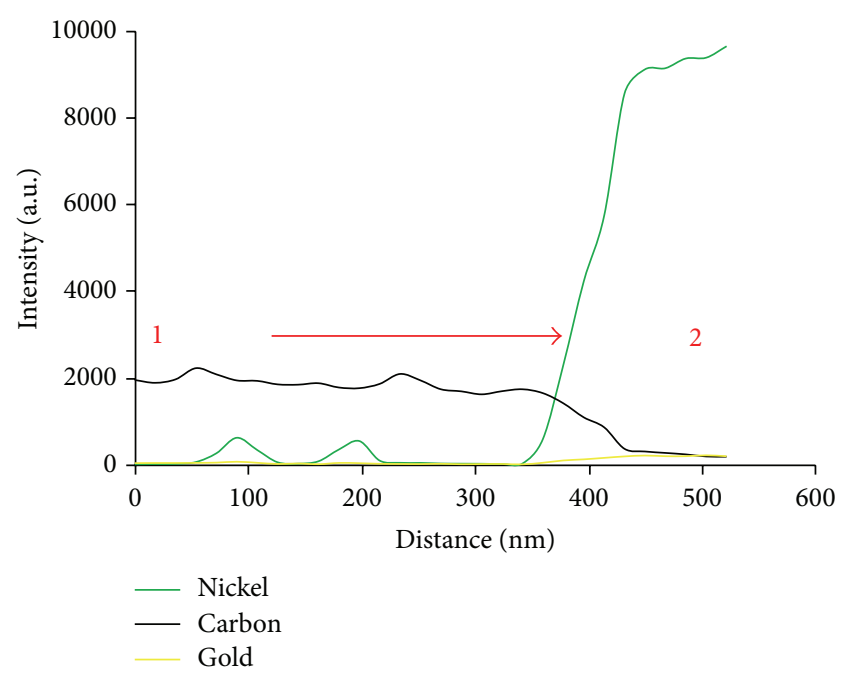

(h)

FIgURE 5: (a-f) TEM images showing the morphology, structure, and interface for NW-CNT heterostructures and (g and h) STEM mode EDS line profile showing NW-CNT heterostructure. The red arrow indicates the direction of line-profiling scan and the location.

$3-5 \mathrm{~cm}^{-1}$ spectral resolution in the range of $1500-1800 \mathrm{~cm}^{-1}$ with $10 \mathrm{~mW}$ laser power. A rectangular grid of $15 \mu \mathrm{m} \times 15 \mu \mathrm{m}$ of the sample surface was chosen and scanned 100 points in full range.

\section{Results and Discussion}

Figure 1 shows the schematic of the heterostructures fabrication: (a) electrodeposition of $\mathrm{Au} / \mathrm{Ni}$ multisegment nanowires 


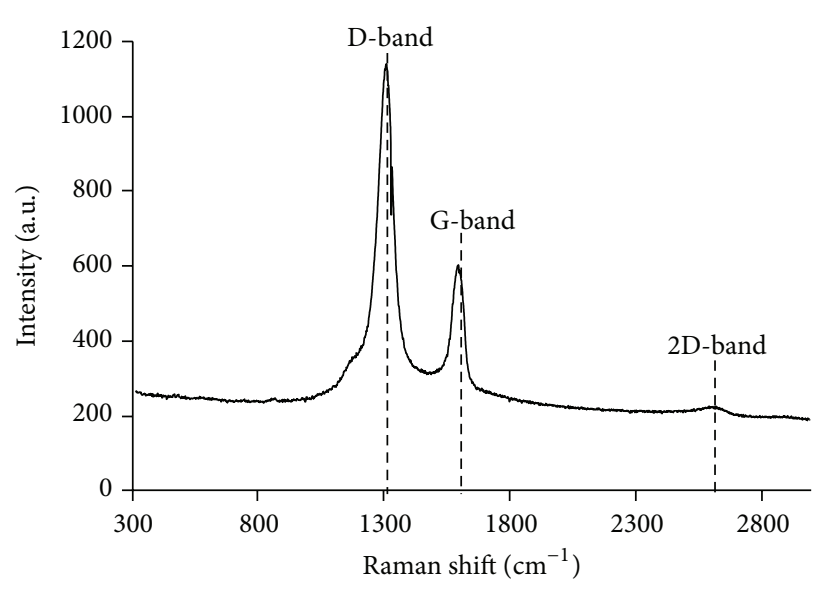

(a)

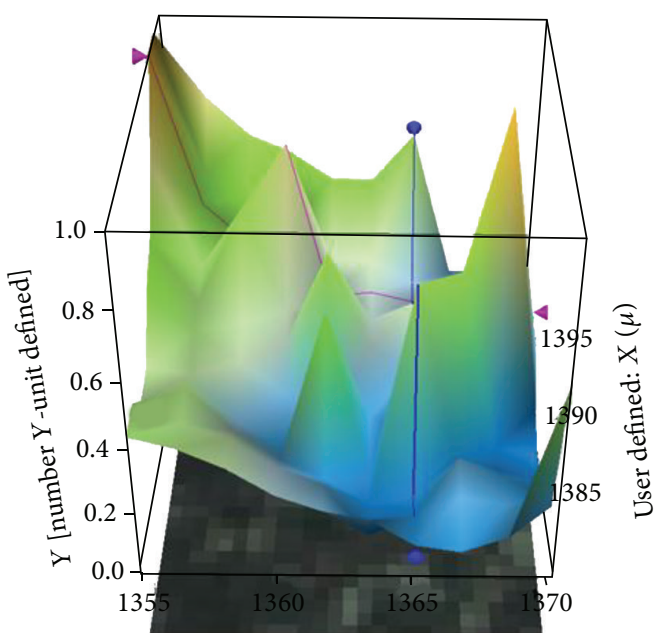

(b)

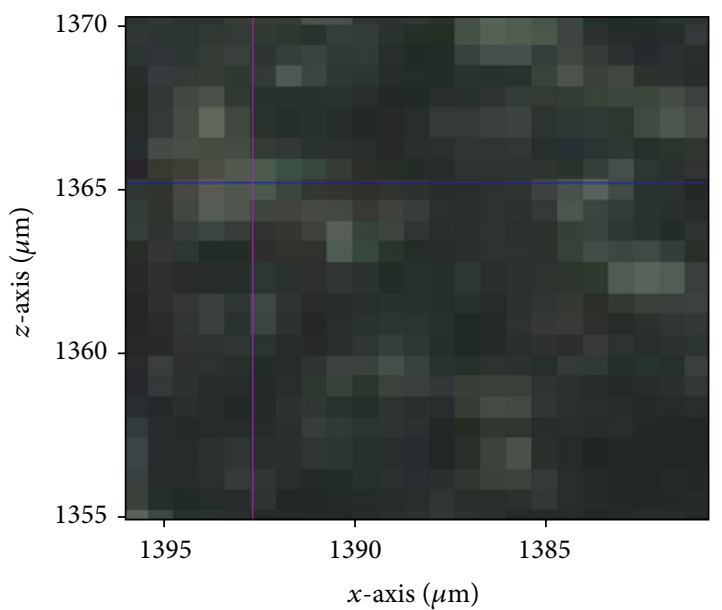

(c)

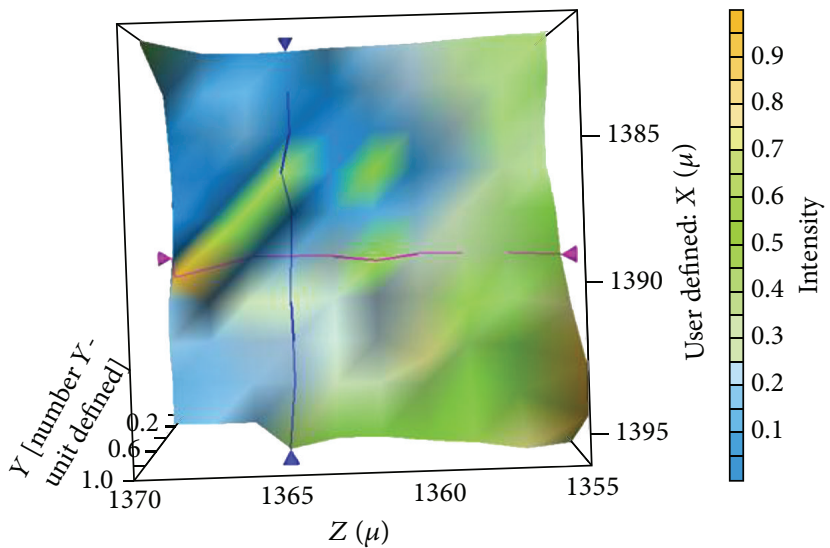

(d)

FIGURE 6: (a) Raman spectrum of NW-CNT heterostructures. (b) 3D chemical mapping for G-band for the heterostructures. (c) Optical image at 100x magnification showing sample surface. (d) Vertical view of Raman 3D chemical map.

using AAO as template, (b) growth of multiwalled CNTs in a CVD growth, where $\mathrm{Ni}$ nanowires acted as catalysts and $\mathrm{AAO}$ as a template (this results in NW-CNT heterostructures after the removal of AAO template), and (c) decoration of $\mathrm{Ni}$ nanoparticles on NW-CNT heterostructures in a direct wet-chemical method resulting in Au-Ni nanowires-CNT$\mathrm{Ni}$ nanoparticles heterostructures or NW-CNC heterostructures.

Figure 2 shows the XRD patterns for $\mathrm{Au}-\mathrm{Ni}$ nanowires, NW-CNT heterostructures, and NW-CNC heterostructures. The as-synthesized $\mathrm{Au} / \mathrm{Ni}$ nanowires exhibit $\mathrm{Au}$ peak and strong peaks of Ni ((a) in Figure 2). Nickel exhibited (220), (111), (200), and (311) fcc crystal planes. Au(400) fcc planes were observed for Au-Ni nanowires. The presence of these planes could be attributed to the electrodeposition process $[18,19]$. After CNT growth ((b) in Figure 2), CNT(002) peak was observed [20] and $\mathrm{Ni}_{3} \mathrm{C}(006)$ and $\mathrm{Ni}_{x} \mathrm{C}(009)$ peaks suggested the catalytic $\mathrm{Ni}$ after poisoning during the CVD growth process [21]. After the decoration of Ni nanoparticles on NW-CNT heterostructure, the XRD intensity for $\mathrm{Ni}(111)$ peak was strengthened indicating low energy faceted growth of Ni nanoparticles ((c) in Figure 2). Other peaks for $\mathrm{Au}, \mathrm{Ni}$, and CNTs were also observed for NW-CNC heterostructure samples. Figures 3(a) and 3(b) show the SEM images of wellaligned $\mathrm{Au} / \mathrm{Ni}$ nanowires over large area after being released from AAO template. The EDS spectra (Figure 3(a), inset) clearly show the presence of $\mathrm{Ni}$ and $\mathrm{Au}$ in the multisegment nanowires. The interface between the $\mathrm{Au}$ segment and the Ni segment is also shown in Figure 3(c). Previously [22, 23], multisegment $\mathrm{Ni}-\mathrm{Au}-\mathrm{Ni}$ nanowires were studied for selective protein tagging and nanoresistor applications.

After the electrodeposition of $\mathrm{Au}-\mathrm{Ni}$ nanowires, multiwalled CNTs were grown on tips of $\mathrm{Ni}$ nanowire segment, inside AAO template. Figure 4(a) shows the freestanding NW-CNT heterostructures after the removal of AAO template. Each segment of NW-CNT heterostructures was characterized by SEM and EDS (Figures 4(b) and 4(d)). $\mathrm{Ni}$ nanowires segments acted as catalysts to assist the growth of CNTs in a CVD process. Figures 4(c) and 4(e) clearly show the presence of Ni nanoparticles at the tips of CNTs indicating 

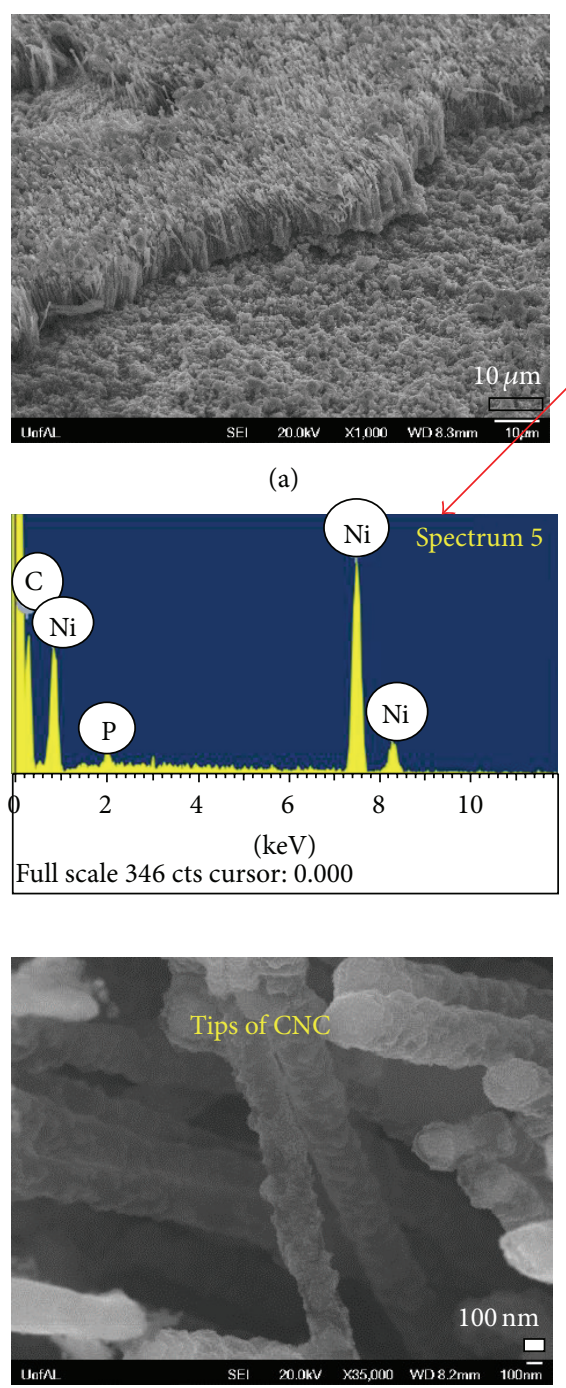

(e)

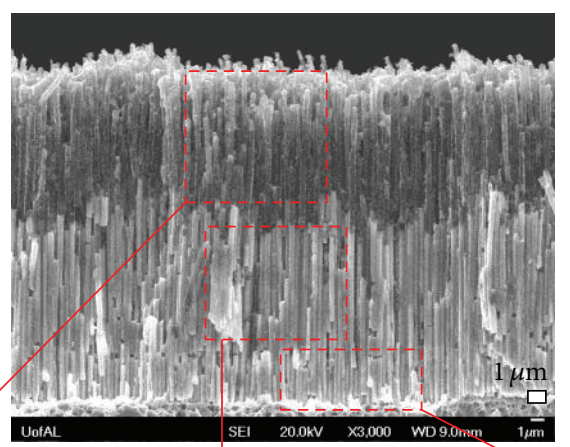

(b)

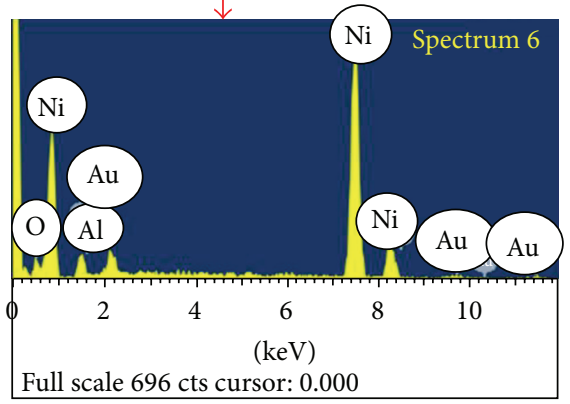

(d)

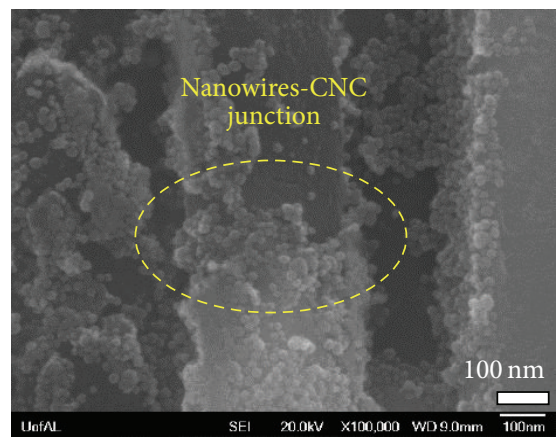

(f)

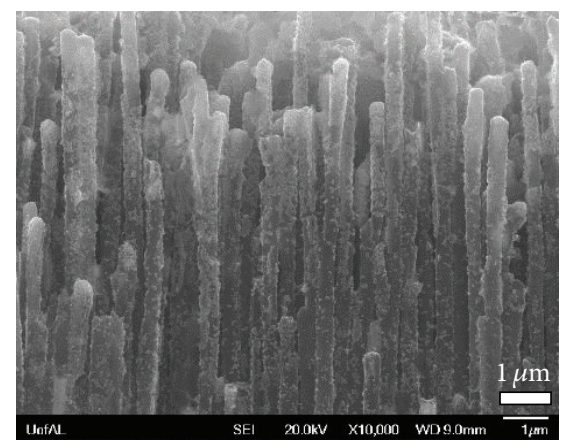

(c)
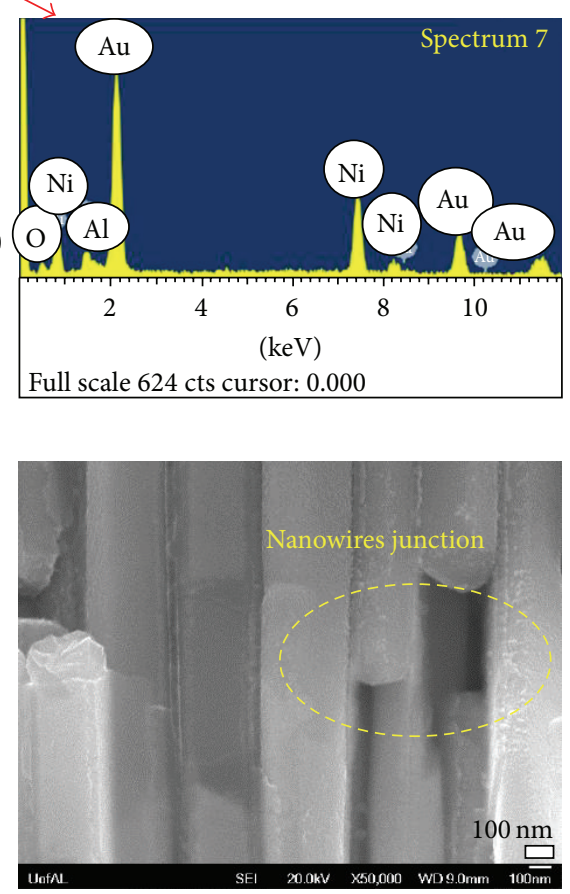

(g)

FIGURE 7: (a-c) SEM images of NW-CNC heterostructures. (d) EDS for different segment of heterostructures, SEM for (e) CNT tips decorated with nanoparticles, (f) heterojunctions for CNT and nanowire segments, and (g) Au-Ni nanowires junctions with some disconnected segments.

tip-growth mechanism for the latter [24]. Nanowires and CNTs were well connected after the CVD growth as shown by the interfaces at the junction (Figure 4(f)). However, for several heterostructures, it was noticed that some Au segments and Ni segments were disconnected after the CVD process (Figure 4(g)). This may be attributed to recrystallization of $\mathrm{Ni}$ or Au nanowires during high temperature CVD process [19, 25]. HR-TEM and EDS line-profiling method were used to characterize NW-CNT heterostructure interfaces (Figure 5). HR-TEM shows the $\sim 0.34 \mathrm{~nm}$ interlayer spacing for CNTs suggesting a graphitic structure [17]. In addition, it is also possible to see the interface with $\mathrm{Ni}$ nanowire segment, where the graphitic carbon is grown parallel to the $\mathrm{Ni}$ nanowire tip and forming junction with the CNT hollow core. This suggests that layered graphitic carbon formation took place at the interface, which was later continued via tip-growth mechanism carrying the initiator Ni nanoparticle along the growing CNT tip. The EDS line-profiling of NWCNT heterostructure interface showed that not only the tips of CNTs contain $\mathrm{Ni}$, but also there was $\mathrm{Ni}$ diffusion across the CNT length as seen by EDS line profile [17]. Furthermore, the NW-CNT heterostructures were characterized by Raman spectroscopy. Figure 6 showed a typical Raman spectrum of NW-CNT heterostructures and chemical mapping for Gband of CNTs for a larger area of standing heterostructures with CNTs facing the incident Raman laser. The peaks for graphite structure including $\mathrm{D}_{-}, \mathrm{G}-$, and 2D-band were clearly observed in the Raman spectrum.

Figure 7 represented SEM images and EDS for NW-CNC heterostructures. It can be observed that, even after the wetchemical nucleation process, the heterostructures remain vertically aligned or standing on the substrate. The EDS analysis shows the presence of various elements on the heterostructures with strong presence of $\mathrm{Ni}$ in different regions 


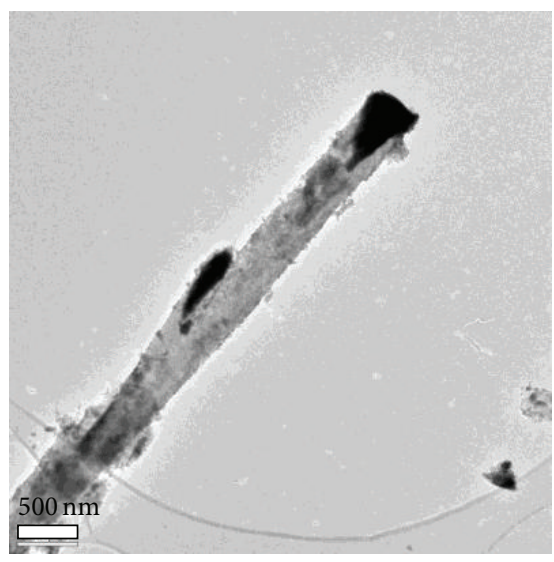

(a)

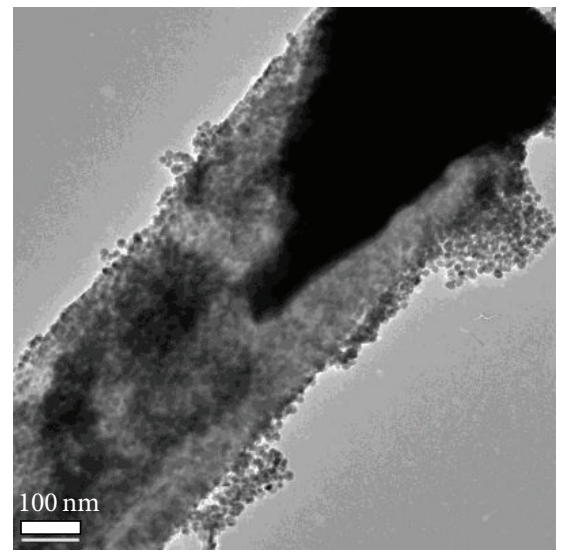

(b)

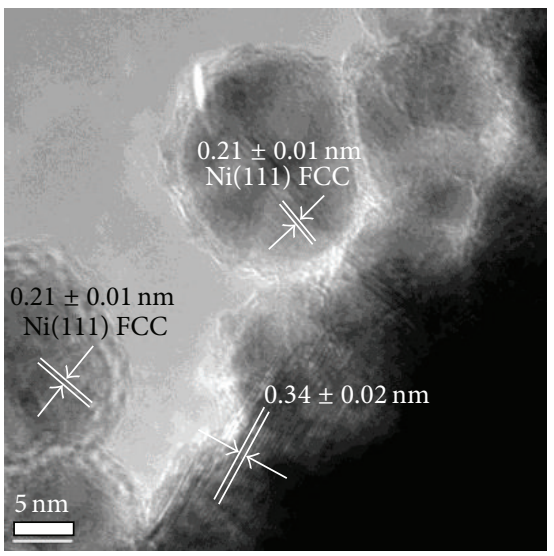

(c)
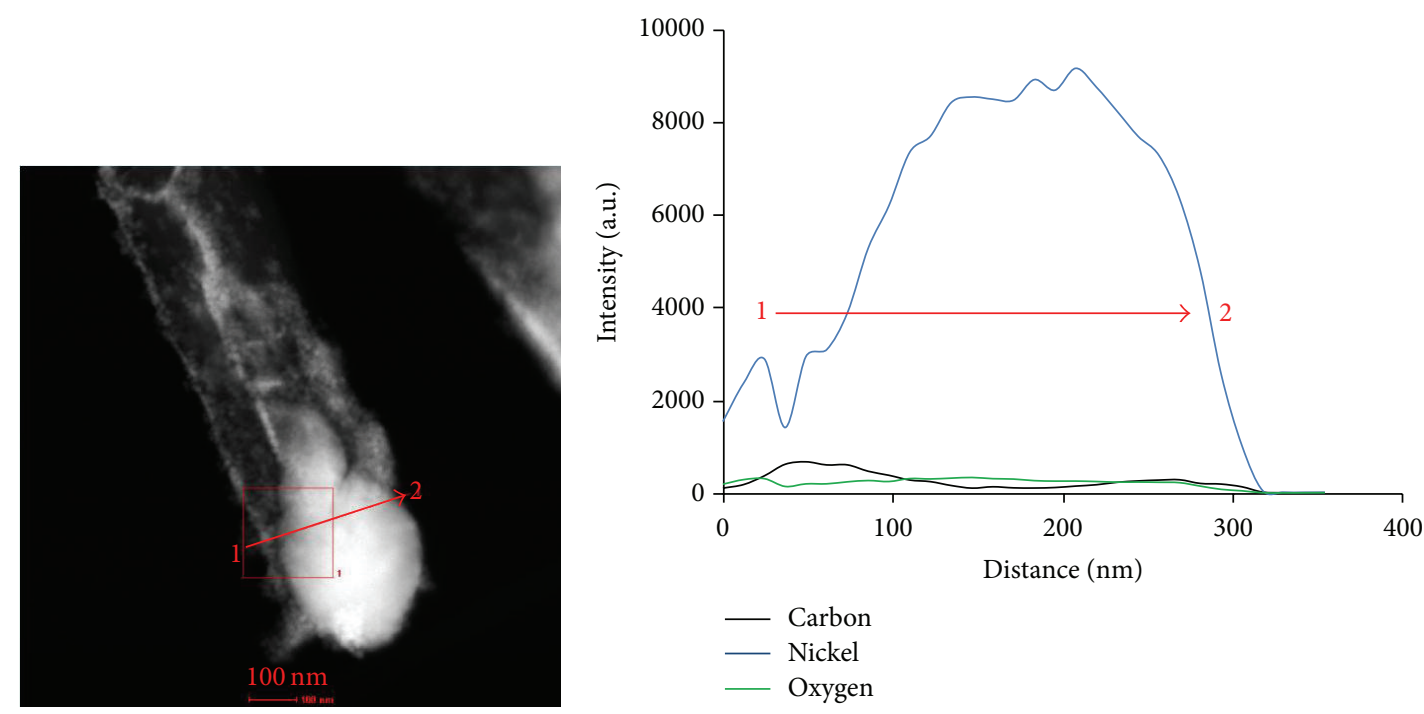

(d)

(e)

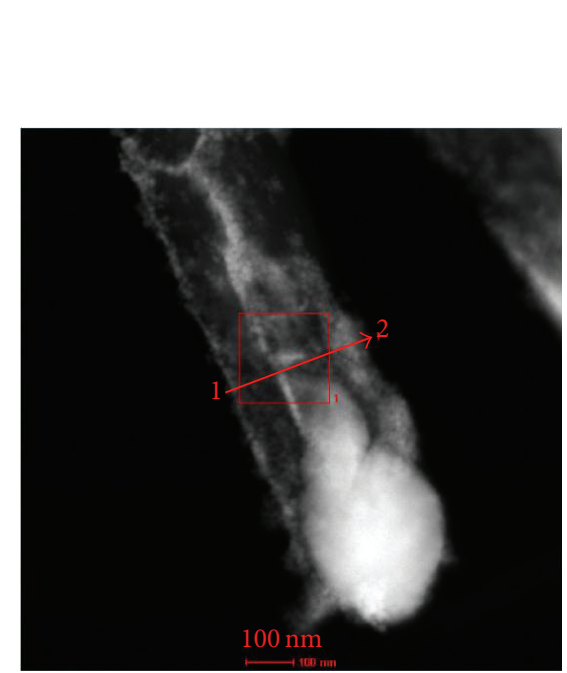

(f)

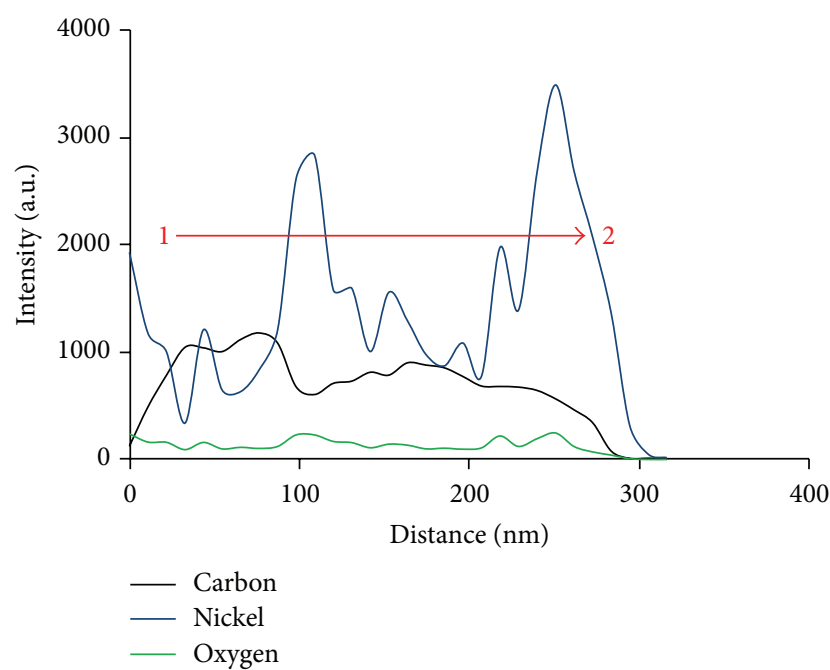

(g)

FIGURE 8: (a-c) TEM images showing NW-CNC heterostructures. (b) The CNT tip with catalyst Ni nanoparticle within the core and decorated with $\mathrm{Ni} / \mathrm{NiO}$ core/shell nanoparticles on the external surface. (c) Core/shell structure of the decorated nanoparticles. (d-g) STEM mode EDS line profile for nanoparticle-decorated CNT tip and the other section of the same CNT. 


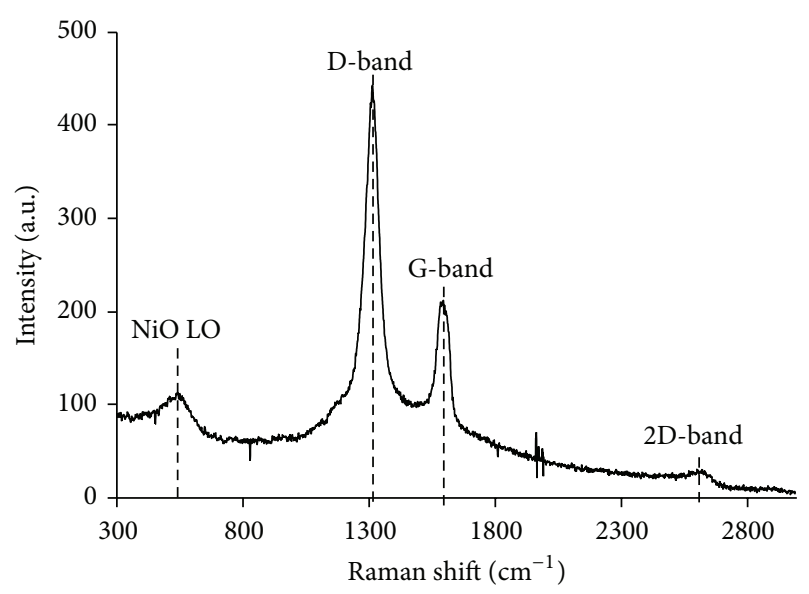

(a)

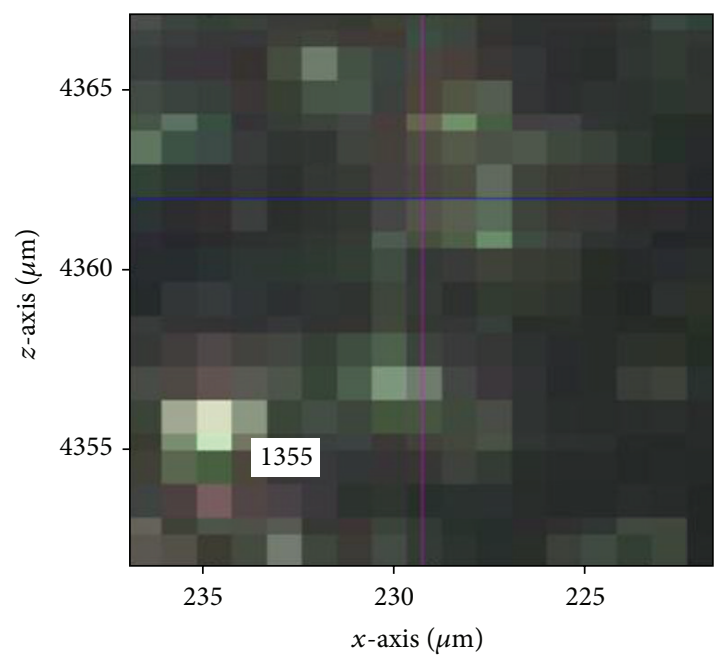

(c)

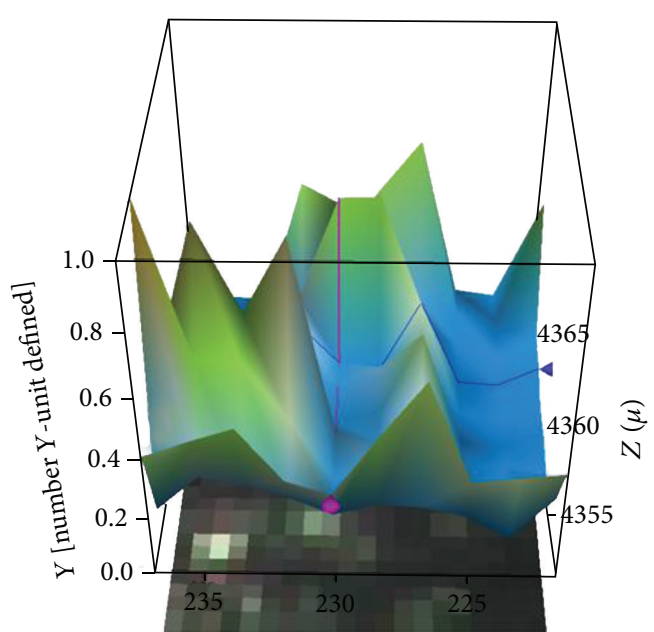

(b)

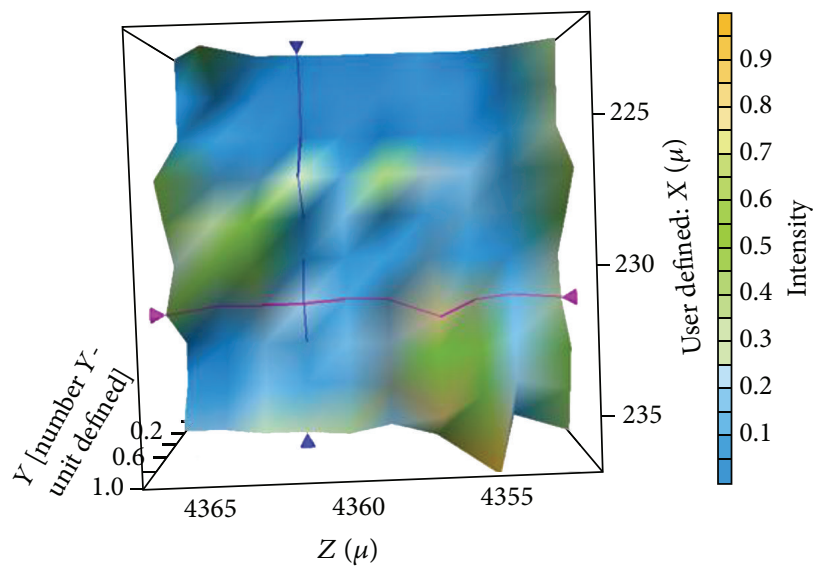

(d)

Figure 9: (a) Raman spectrum for NW-CNC heterostructures. (b) 3D chemical mapping for G-band intensity for NW-CNC heterostructures. (c) Optical image at 100x magnification for sample surface. (d) Vertical view of 3D chemical map.

(Figures 7(b) and 7(d)). This indicates that $\mathrm{Ni}$ was nucleated on all the segments of the heterostructures. However, it could be noticed from the SEM images that the nucleation of $\mathrm{Ni}$ nanoparticles was mostly observed on the CNT segment (Figures $7(\mathrm{c})$ and $7(\mathrm{e})-7(\mathrm{~g})$ ). Ni nanoparticles with diameter $\sim 20 \mathrm{~nm}$ were decorated on NW-CNT heterostructures. The presence of nanoparticles majorly on the surface of CNT was observed due to the good wettability of hydrophobic CNT segment in oil phase solution for nucleation of $\mathrm{Ni}$ nanoparticles (Figures 7(f) and 7(g)) [17]. TEM and EDS line-profiling for NW-CNC heterostructures are shown in Figure 8. Consistent with SEM observations, majority of $\mathrm{Ni}$ nanoparticles were present on CNT segment of heterostructures (Figures 8(a) and 8(b)). HR-TEM of decorated $\mathrm{Ni}$ nanoparticles (Figure 8(c)) indicated that single-crystal Ni nanoparticles were encapsulated in thin polycrystalline $\mathrm{NiO}$ shells attributed to air exposure of heterostructures [26]. Furthermore, scanning transmission electron microscopy (STEM) mode EDS line profiles are performed along the diameter of nanoparticle-decorated CNT segments (Figures $8(\mathrm{~d})-8(\mathrm{~g}))$. This clearly shows the Ni nanoparticle decoration. To differentiate between the signal from Ni nanoparticle (CNT growth catalyst) inside the CNT core and $\mathrm{Ni}$ nanoparticles decorated by wet-chemical approach, the line profiles were performed in two different regions of CNTs as shown by Figures 8(d) and 8(f). The broad Ni peak (blue color) in Figure 8(e) is due to the $\mathrm{Ni}$ nanoparticle within the CNT core, while Figure 8(g) clearly shows the multiple peaks of $\mathrm{Ni}$ across the CNT diameter. Uniform peaks of $\mathrm{C}$ and $\mathrm{O}$ are also observed, where the latter can be attributed to the $\mathrm{NiO}$ shell around $\mathrm{Ni}$ nanoparticles. The formation of $\mathrm{NiO}$ on $\mathrm{Ni}$ nanoparticles was further confirmed by Raman shift peaks for longitudinal optical phonon modes (LO, $546 \mathrm{~cm}^{-1}$ ) in Raman spectrum for NWCNC heterostructures (Figure 9(a)) [27]. Figures 9(b)-9(d) show the chemical maps of the heterostructures. Knowing the density of nanowires $\left(\sim 8.3 \times 10^{8} \mathrm{~cm}^{-2}\right)$, the length and diameter of nanowires and CNTs, and the diameter of $\mathrm{Ni}$ 


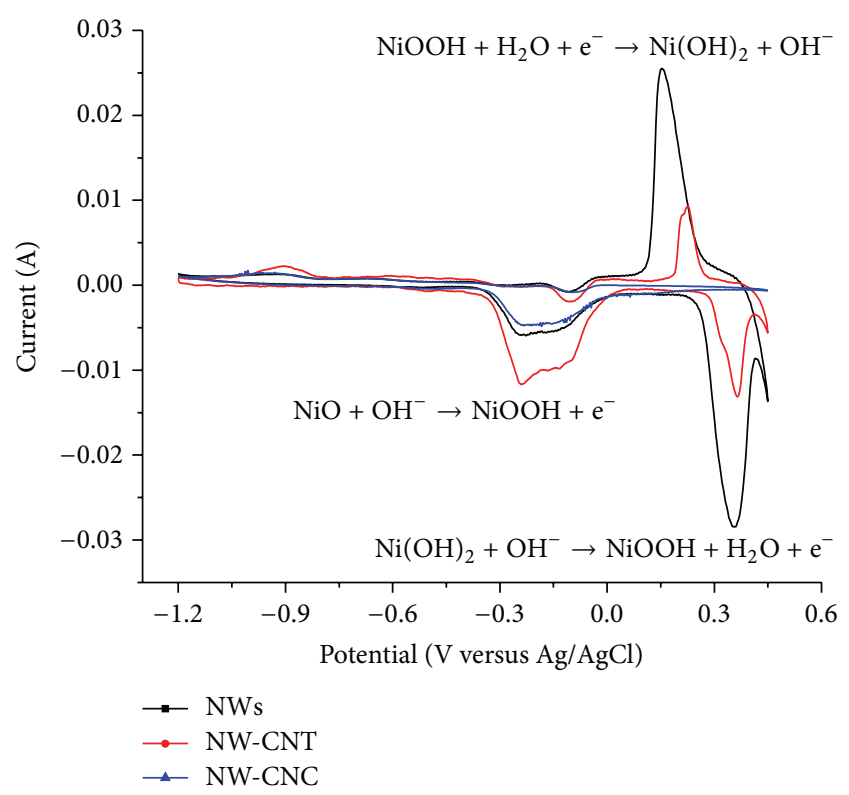

FIGURE 10: Representative cyclic voltammetric (CV) measurements for NWs, NW-CNT heterostructures, and NW-CNC heterostructures in $9 \mathrm{M} \mathrm{KOH}$ solution with scan rate of $30 \mathrm{mV} / \mathrm{s}$.

nanoparticles, it was possible to calculate the specific area of $\mathrm{Au}-\mathrm{Ni}$ nanowires, NW-CNT heterostructures, and NWCNC heterostructures (see Table S1 in Supplementary Material available online at http://dx.doi.org/10.1155/2015/125970). This shows that the highest surface area is observed for NWCNT heterostructures and the lowest for after decoration with nanoparticles for the case of NW-CNC heterostructures. The latter could be due to the aggregation of nucleated nanoparticles. These are only rough estimates of surface area and thus could be lower than actual values and would be a critical factor for applications of such heterostructures such as supercapacitors [28].

The carbon-based active materials that have high surface areas are commonly used in electrochemical doublelayer capacitors (EDLCs). Another group of electrochemical capacitors (ECs) utilizes fast and reversible surface or nearsurface reactions for charge storage, known as pseudocapacitors or redox supercapacitors which are also combined with EDLCs or battery electrode to form hybrid capacitors. Among various pseudocapacitor materials nickel oxide is known to be promising [29]. CNTs have proven to be good materials to construct supercapacitor electrodes due to the high conductivity, electrochemical stability, and open porosity [30]. So the construction of Au-Ni NWs-CNTs hybrid structures holds potential as capacitors. The Au segment was designed as current collector [10], Ni was selected as pseudocapacitor materials [31] or catalyst for the growth of CNTs [32], and CNTs would impart double-layer characteristics.

The capacitive behavior of an electrode material is generally characterized by cyclic voltammogram (CV). In an ideal electrical double-layer capacitor containing smooth electrodes, its CV current response shows a rectangular mirror image with respect to the zero-current line [33]. When faradic reaction and Ohmic resistance (resulting from electrolyte diffusion within porous electrode) are involved, the rectangular mirror image is no longer maintained [34]. The capacitance can be calculated through the following equations [35]:

$$
\begin{gathered}
C=\frac{q}{V_{f}-V_{i o}}, \\
q=\frac{\int I(V) d V}{v_{\text {scan }}},
\end{gathered}
$$

where $C$ is capacitance $(\mathrm{F}), q$ is quantity of electric charge (C), $V_{f}$ is final potential, $V_{i o}$ is starting potential, $\int I(V) d V$ is the area of CV curve and represents the average, and $v_{\text {scan }}$ is the potential scan rate for $\mathrm{CV}$ test. The faradic capacitance is due to the $\mathrm{Ni}^{2+}$ to $\mathrm{Ni}^{3+}$ redox reaction given as $[36,37]$ :

$$
\mathrm{NiO}+\mathrm{OH}^{-} \longleftrightarrow \mathrm{NiOOH}+\mathrm{e}^{-}
$$

Nickel hydroxides also exist in the $\mathrm{Ni}^{2+}$ to $\mathrm{Ni}^{3+}$ redox couple in alkaline solution as $[34,36]$ :

$$
\mathrm{Ni}(\mathrm{OH})_{2}+\mathrm{OH}^{-} \longleftrightarrow \mathrm{NiOOH}+\mathrm{H}_{2} \mathrm{O}+\mathrm{e}^{-}
$$

A typical CV measurement for NWs, NW-CNT heterostructures, and NW-CNC heterostructures is shown in Figure 10. Capacitance for each sample at different conditions was calculated and listed in Table S2 (Supporting Information). The capacitance in NWs could be attributed to the presence of thin $\mathrm{NiO}$ layer around $\mathrm{Ni}$ segments or the metallic segment double-layer formation characteristics. However, the oxide layer on $\mathrm{Ni}$ segment was indiscernible in electron microscopic characterizations. Generally, a low scan rate, high $\mathrm{KOH}$ concentration, and suitable scan range/window can enhance the electron transfer reactions on electrode, thus resulting in higher capacitance (Figure S1 and S2, Table S2 in Supporting Information) [38]. Figure 11 shows the trends for the specific capacitance for the NWs, NW-CNT heterostructures, and $\mathrm{NW}-\mathrm{CNC}$ heterostructures. It was observed that specific capacitance decreased with increasing electrolyte concentration, decreasing scan window, and increasing scan rates. The systematic studies performed here are of great value for understanding and developing optimized energy storage devices using such heterostructures. It is also noted that after adding CNT and Ni nanoparticles on the heterostructures, the capacitance was lowered as compared to other samples. This may be attributed to two reasons: (1) After CVD growth of CNT and Ni nanoparticles nucleation, some heterojunctions between $\mathrm{Au}-\mathrm{Ni}$ nanowires were damaged and disconnected as observed in Figures 4(g) and $7(\mathrm{~g})$. The disconnection of nanowires would interrupt the current collection step when those materials were measured with CV. (2) The surface of multiwalled CNTs grown from AAO template by CVD is hydrophobic. The wettability of CNTs would significantly affect the exposed area of electrode; thus a hydrophobic surface may reduce the exposed area of electrode with solution [39]. Also, the Ni nanoparticles nucleation step was conducted in oil phase solution, which may have led to contamination of hydrophobic surfactant species on the heterostructures. The hydrophobic surface 


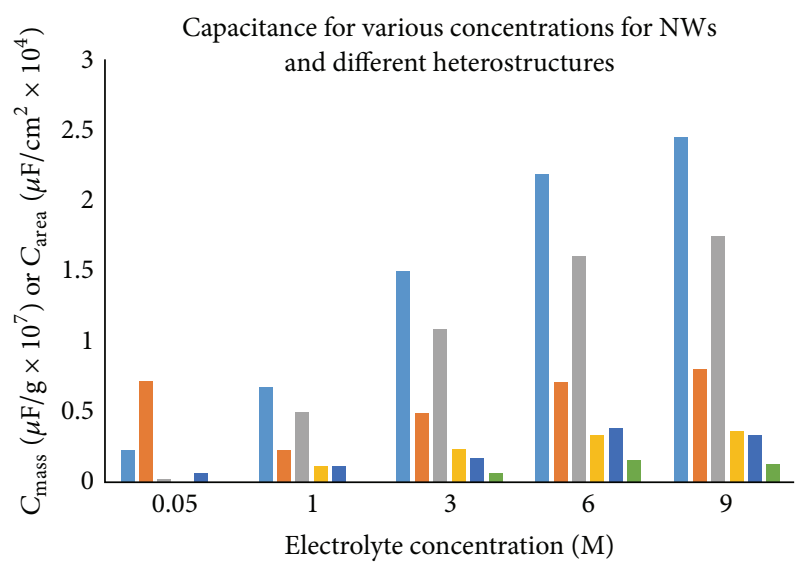

(a)

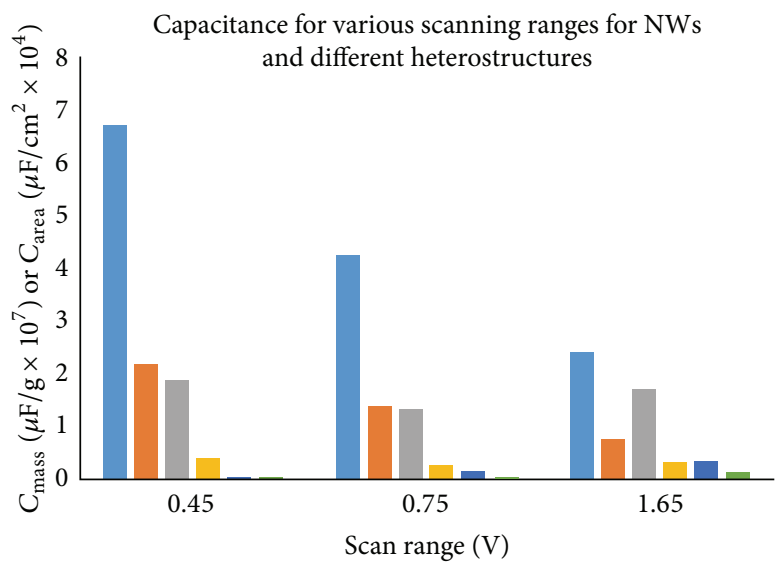

(b)

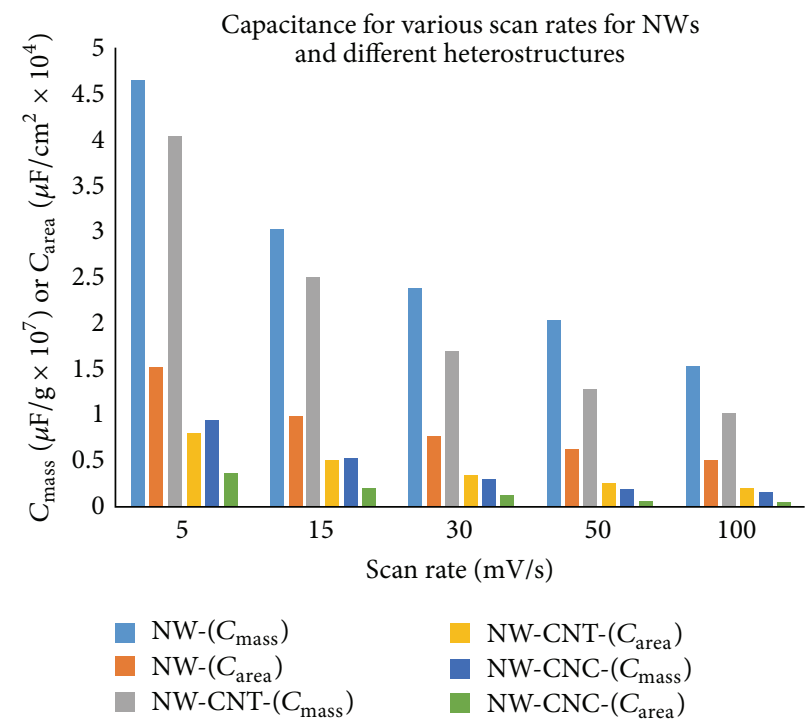

(c)

FIGURE 11: Specific capacitance versus electrolyte concentration, scan range, and scan rate for various samples (NWs, NW-CNT heterostructures, and NW-CNC heterostructures). The capacitance values correspond to with respect to mass $\left(C_{\text {mass }}\right)$ and area $\left(C_{\text {area }}\right)$.

would inhibit the redox reaction since the $\mathrm{KOH}$ solution cannot properly wet the nanostructures. Thus, the surface wettability of NW-CNT and NW-CNC heterostructures must be tuned in order to achieve higher capacitance values.

\section{Conclusions}

Vertically aligned Au-Ni nanowires axially linked with CNTs with uniform diameters and micron-scale lengths were fabricated by combining electrodeposition and CVD methods by using porous alumina or anodized aluminum oxide template (AAO). Furthermore, these NW-CNT heterostructures were integrated with $\mathrm{Ni} / \mathrm{NiO}$ core/shell nanoparticles in a wetchemical approach. The diameters of nanowires and CNTs were determined by porous template size and lengths were controlled by varying the duration of electrodeposition and CVD process. The structural, interfacial, and morphological characterization were performed using microscopic and spectroscopic methods. $\mathrm{Ni}$ segment of $\mathrm{Au}-\mathrm{Ni}$ nanowires was utilized as a catalyst for the CVD growth of CNTs.
However, it was observed that CVD growth process results in disconnected $\mathrm{Au}$ and $\mathrm{Ni}$ segments due to material migration. Overall, the CVD processing for CNT growth and wetchemical nucleation of nanoparticles did not disturb the vertical alignment of heterostructures. Electrochemical behavior of various heterostructures was studied and compared. NWCNT heterostructures showed the highest surface area. The specific capacitance of NWs, NW-CNT heterostructures, and $\mathrm{NW}-\mathrm{CNC}$ heterostructures was investigated as a function of electrolyte $(\mathrm{KOH})$ concentration, scan rate, and scan voltage range. The highest specific capacitance for $\mathrm{Au} / \mathrm{Ni} \mathrm{NWs}$ was around $6.8 \times 10^{7} \mu \mathrm{F} / \mathrm{g}$. However, the NW-CNT and NW$\mathrm{CNC}$ heterostructures showed the highest capacitance of $\sim$ $4.1 \times 10^{7} \mu \mathrm{F} / \mathrm{g}$ and $9.7 \times 10^{6} \mu \mathrm{F} / \mathrm{g}$, respectively. This decrease in specific capacitance as compared to $\mathrm{Au} / \mathrm{Ni} \mathrm{NWs}$ could be attributed to the damaged interfaces for the nanowire segments and the hydrophobicity of CNTs or hydrophobic contamination of heterostructures during wet-chemical nucleation and growth of Ni nanoparticles. 


\section{Conflict of Interests}

The authors declare that there is no conflict of interests regarding the publication of this paper.

\section{Authors' Contribution}

The project was conceptualized and designed by Nitin Chopra, while experiments were done by Paaras Agrawal and Junchi Wu.

\section{Acknowledgments}

This work has been supported by The University of Alabama's Research Grant Committee Award, NSF-REH award through MINT Center, and NSF-EPSCoR RII award. P. S. thanks NSF-REH for the summer internship financial support. The authors also thank the Central Analytical Facility (CAF) for electron microscopy equipment and other centers for providing infrastructure support such as clean room facility and various kinds of equipment. Nitin Chopra wrote the paper and conducted the analysis.

\section{References}

[1] S. J. Hurst, E. K. Payne, L. Qin, and C. A. Mirkin, "Multisegmented one-dimensional nanorods prepared by hard-template synthetic methods," Angewandte Chemie, vol. 45, no. 17, pp. 2672-2692, 2006.

[2] N. Chopra, "Multifunctional and multicomponent heterostructured one-dimensional nanostructures: advances in growth, characterisation, and applications," Materials Technology, vol. 25, no. 3-4, pp. 212-230, 2010.

[3] F. S. Ou, M. M. Shaijumon, and P. M. Ajayan, "Controlled manipulation of giant hybrid inorganic nanowire assemblies," Nano Letters, vol. 8, no. 7, pp. 1853-1857, 2008.

[4] B. J. Hinds, N. Chopra, T. Rantell, R. Andrews, V. Gavalas, and L. G. Bachas, "Aligned multiwalled carbon nanotube membranes," Science, vol. 303, no. 5654, pp. 62-65, 2004.

[5] R. Andrews, D. Jacques, A. M. Rao et al., "Continuous production of aligned carbon nanotubes: a step closer to commercial realization," Chemical Physics Letters, vol. 303, no. 5-6, pp. 467474, 1999.

[6] S. Fan, M. G. Chapline, N. R. Franklin, T. W. Tombler, A. M. Cassell, and H. Dai, "Self-oriented regular arrays of carbon nanotubes and their field emission properties," Science, vol. 283, no. 5401, pp. 512-514, 1999.

[7] J. T. Hu, M. Ouyang, P. D. Yang, and C. M. Lieber, "Controlled growth and electrical properties of heterojunctions of carbon nanotubes and silicon nanowires," Nature, vol. 399, no. 6731, pp. 48-51, 1999.

[8] J. Luo, J. Zhu, Z. Huang, and L. Zhang, "Arrays of Ni nanowire/ multiwalled carbon nanotube/amorphous carbon nanotube heterojunctions containing Schottky contacts," Applied Physics Letters, vol. 90, Article ID 033114, 2007.

[9] G. L. Che, B. B. Lakshmi, E. R. Fisher, and C. R. Martin, "Carbon nanotubule membranes for electrochemical energy storage and production," Nature, vol. 393, no. 6683, pp. 346-349, 1998.

[10] K. H. An, W. S. Kim, Y. S. Park et al., "Electrochemical properties of high-power supercapacitors using single-walled carbon nanotube electrodes," Advanced Functional Materials, vol. 11, no. 5, pp. 387-392, 2001.

[11] A. Burke, "Ultracapacitors: why, how, and where is the technology," Journal of Power Sources, vol. 91, no. 1, pp. 37-50, 2000.

[12] M. M. Shaijumon, F. S. Ou, L. J. Ci, and P. M. Ajayan, "Synthesis of hybrid nanowire arrays and their application as high power supercapacitor electrodes," Chemical Communications, vol. 20, pp. 2373-2375, 2008.

[13] A. G. Pandolfo and A. F. Hollenkamp, "Carbon properties and their role in supercapacitors," Journal of Power Sources, vol. 157, no. 1, pp. 11-27, 2006.

[14] K. W. Nam, W. S. Yoon, and K. B. Kim, "X-ray absorption spectroscopy studies of nickel oxide thin film electrodes for supercapacitors," Electrochimica Acta, vol. 47, no. 19, pp. 32013209, 2002

[15] J. Y. Lee, K. Liang, K. H. An, and Y. H. Lee, "Nickel oxide/carbon nanotubes nanocomposite for electrochemical capacitance," Synthetic Metals, vol. 150, no. 2, pp. 153-157, 2005.

[16] D.-W. Wang, F. Li, and H.-M. Cheng, "Hierarchical porous nickel oxide and carbon as electrode materials for asymmetric supercapacitor," Journal of Power Sources, vol. 185, no. 2, pp. 1563-1568, 2008.

[17] N. Chopra, W. W. Shi, and A. Bansal, "Structural evolution and stability studies of heterostructures comprised of carbon nanotubes decorated with nickel/nickel oxide core/shell nanoparticles," Carbon, vol. 49, no. 11, pp. 3645-3662, 2011.

[18] T. K. Sau and A. L. Rogach, "Nonspherical noble metal nanoparticles: colloid-chemical synthesis and morphology control," Advanced Materials, vol. 22, no. 16, pp. 1781-1804, 2010.

[19] H. Pan, H. Sun, C. Poh, Y. P. Feng, and J. Y. Lin, "Single-crystal growth of metallic nanowires with preferred orientation," Nanotechnology, vol. 16, no. 9, pp. 1559-1564, 2005.

[20] L. J. Ci, B. Q. Wei, C. L. Xu et al., "Crystallization behavior of the amorphous carbon nanotubes prepared by the CVD method," Journal of Crystal Growth, vol. 233, no. 4, pp. 823-828, 2001.

[21] W. Zhou, K. Zheng, L. He et al., "Ni/ $/ \mathrm{Ni}_{3} \mathrm{C}$ core-shell nanochains and its magnetic properties: one-step synthesis at low temperature," Nano Letters, vol. 8, no. 4, pp. 1147-1152, 2008.

[22] K.-B. Lee, S. Park, and C. A. Mirkin, "Multicomponent magnetic nanorods for biomolecular separations," Angewandte Chemie International Edition, vol. 43, no. 23, pp. 3048-3050, 2004.

[23] C. M. Hangarter and N. V. Myung, "Magnetic alignment of nanowires," Chemistry of Materials, vol. 17, no. 6, pp. 1320-1324, 2005.

[24] J. Han, J. B. Yoo, C. Y. Park et al., "Tip growth model of carbon tubules grown on the glass substrate by plasma enhanced chemical vapor deposition," Journal of Applied Physics, vol. 91, no. 1, pp. 483-486, 2002.

[25] M. J. Luton and C. M. Sellars, "Dynamic recrystallization in nickel and nickel-iron alloys during high temperature deformation," Acta Metallurgica, vol. 17, no. 8, pp. 1033-1043, 1969.

[26] X.-H. Xia, J.-P. Tu, X.-L. Wang, C.-D. Gu, and X.-B. Zhao, "Hierarchically porous $\mathrm{NiO}$ film grown by chemical bath deposition via a colloidal crystal template as an electrochemical pseudocapacitor material," Journal of Materials Chemistry, vol. 21, no. 3, pp. 671-679, 2011.

[27] W. Z. Wang, Y. K. Liu, C. K. Xu, C. L. Zheng, and G. L. Wang, "Synthesis of $\mathrm{NiO}$ nanorods by a novel simple precursor thermal decomposition approach," Chemical Physics Letters, vol. 362, no. 1-2, pp. 119-122, 2002. 
[28] P. Simon and Y. Gogotsi, "Materials for electrochemical capacitors," Nature Materials, vol. 7, no. 11, pp. 845-854, 2008.

[29] J.-W. Lang, L.-B. Kong, W.-J. Wu, Y.-C. Luo, and L. Kang, "Facile approach to prepare loose-packed $\mathrm{NiO}$ nano-flakes materials for supercapacitors," Chemical Communications, no. 35, pp. 4213-4215, 2008.

[30] D. N. Futaba, K. Hata, T. Yamada et al., "Shape-engineerable and highly densely packed single-walled carbon nanotubes and their application as super-capacitor electrodes," Nature Materials, vol. 5, no. 12, pp. 987-994, 2006.

[31] G.-H. Yuan, Z.-H. Jiang, A. Aramata, and Y.-Z. Gao, "Electrochemical behavior of activated-carbon capacitor material loaded with nickel oxide," Carbon, vol. 43, no. 14, pp. 2913-2917, 2005.

[32] J. Luo, L. Zhang, Y. J. Zhang, and J. Zhu, "Controlled growth of one-dimensional metal-semiconductor and metal-carbon nanotube heterojunctions," Advanced Materials, vol. 14, no. 19, pp. 1413-1414, 2002.

[33] S. Yoon, J. Lee, T. Hyeon, and S. M. Oh, "Electric double-layer capacitor performance of a new mesoporous carbon," Journal of the Electrochemical Society, vol. 147, no. 7, pp. 2507-2512, 2000.

[34] M.-S. Wu and H.-H. Hsieh, "Nickel oxide/hydroxide nanoplatelets synthesized by chemical precipitation for electrochemical capacitors," Electrochimica Acta, vol. 53, no. 8, pp. 3427-3435, 2008.

[35] V. Srinivasan and J. W. Weidner, "Studies on the capacitance of nickel oxide films: effect of heating temperature and electrolyte concentration," Journal of the Electrochemical Society, vol. 147, no. 3, pp. 880-885, 2000.

[36] C.-T. Hsieh, Y.-W. Chou, and W.-Y. Chen, "Synthesis and electrochemical characterization of carbon nanotubes decorated with nickel nanoparticles for use as an electrochemical capacitor," Journal of Solid State Electrochemistry, vol. 12, no. 6, pp. 663-669, 2008.

[37] V. Srinivasan and J. W. Weidner, "An electrochemical route for making porous nickel oxide electrochemical capacitors," Journal of the Electrochemical Society, vol. 144, no. 8, pp. L210L213, 1997.

[38] R. S. Nicholson, "Theory and application of cyclic voltammetry for measurement of electrode reaction kinetics," Analytical Chemistry, vol. 37, no. 11, pp. 1351-1355, 1965.

[39] W. Shi, K. Venkatachalam, V. Gavalas et al., "The role of plasma treatment on electrochemical capacitance of undoped and nitrogen doped carbon nanotubes," Nanomaterials and Energy, vol. 2, no. 2, pp. 71-81, 2013. 

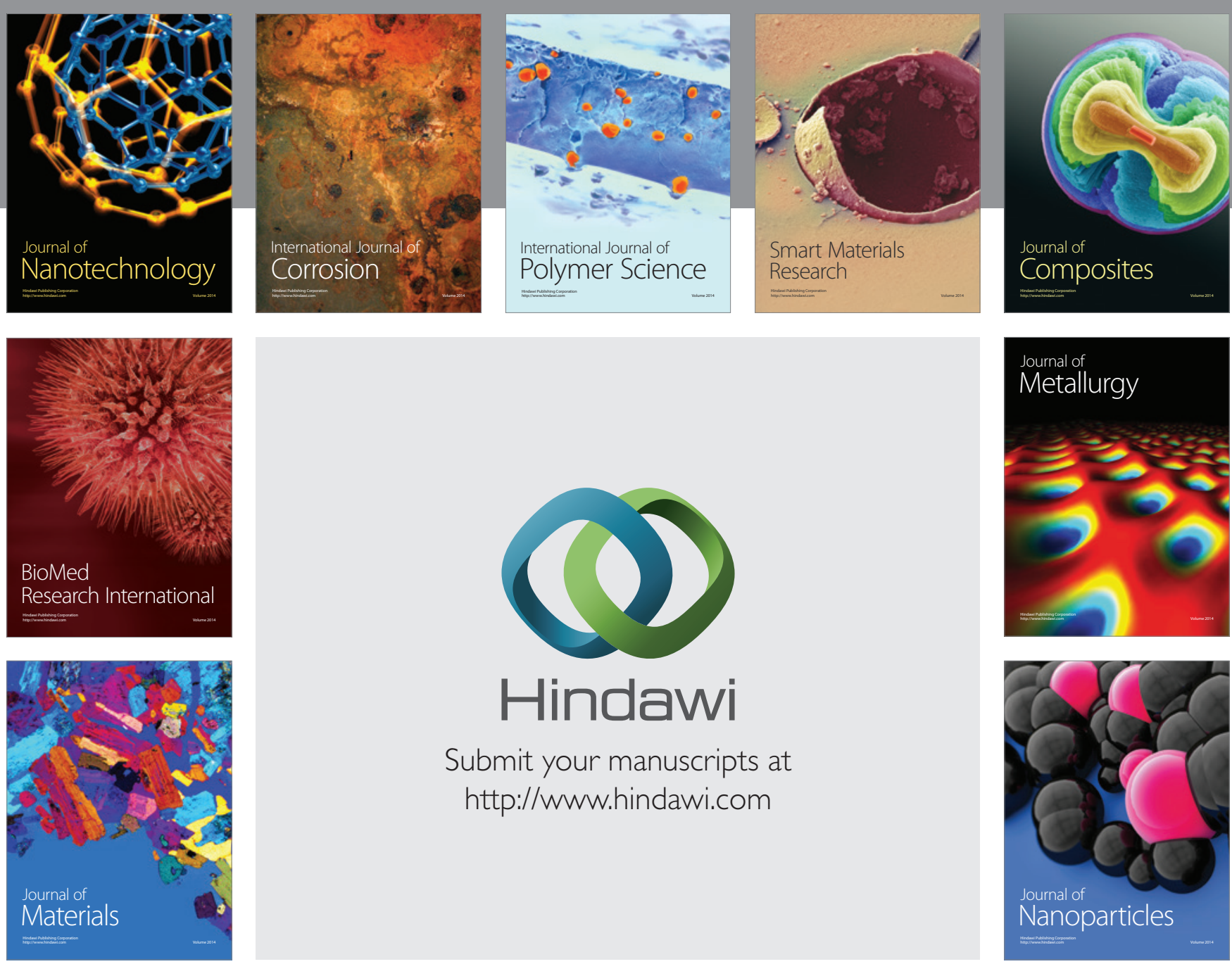

Submit your manuscripts at http://www.hindawi.com
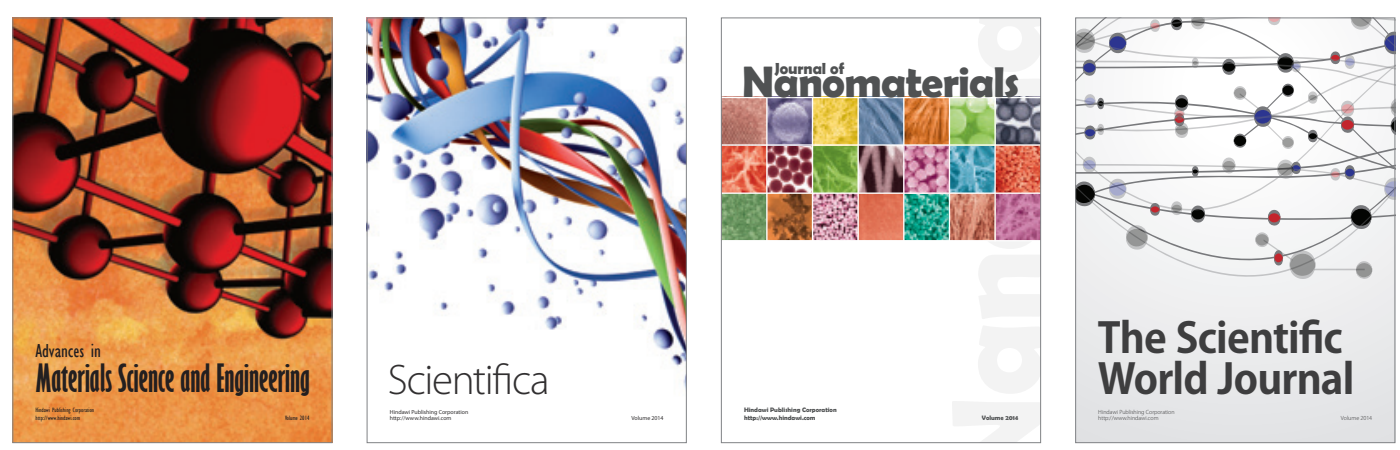

\section{The Scientific World Journal}
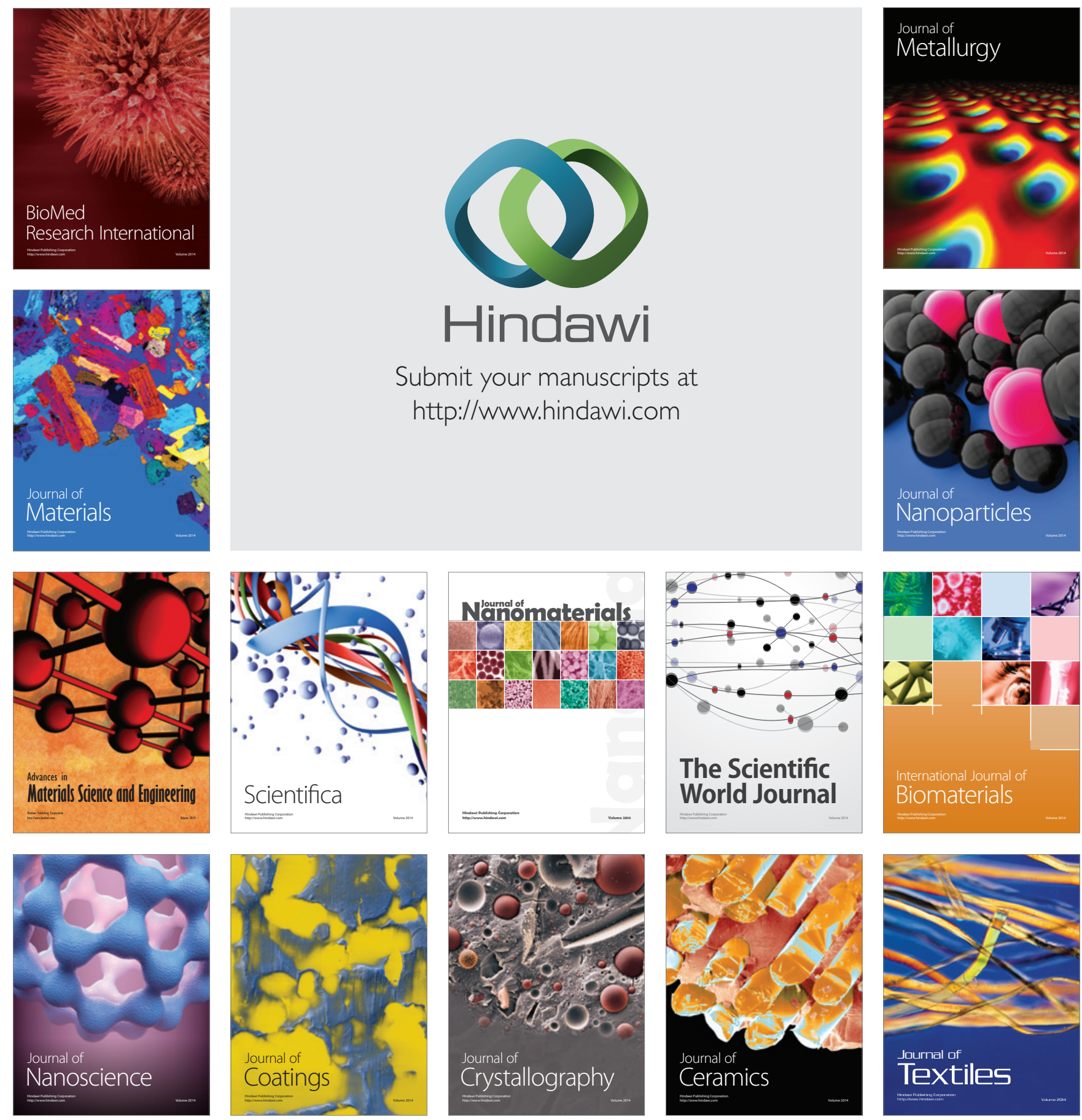